\title{
THE EXISTENCE OF NON-TOPOLOGICAL MULTIVORTEX SOLUTIONS IN THE RELATIVISTIC SELF-DUAL CHERN-SIMONS THEORY
}

\author{
Dongho Chae and Oleg Yu. Imanuvilov
}

\begin{abstract}
We construct a general type of multivortex solutions of the selfduality equations (the Bogomol'nyi equations) of $(2+1)$ dimensional relativistic Chern-Simons model with the non-topological boundary condition near infinity. For such construction we use a modified version of the Newton iteration method developed by Kantorovich.
\end{abstract}

\section{INTRODUCTION}

The Lagrangian density of the (2+1)-dimensional relativistic Chern-Simons gauge field theory is given by

$$
\mathcal{L}=\frac{\kappa}{4} \varepsilon^{\mu \nu \rho} F_{\mu \nu} A_{\rho}+\left(D_{\mu} \phi\right) \overline{\left(D^{\mu} \phi\right)}-\frac{1}{\kappa^{2}}|\phi|^{2}\left(1-|\phi|^{2}\right)^{2},
$$

where $A_{\mu}(\mu=0,1,2)$ is the gauge field on $\mathbb{R}^{3}, F_{\mu \nu}=\frac{\partial}{\partial x^{\mu}} A_{\nu}-\frac{\partial}{\partial x^{\nu}} A_{\mu}$ is the corresponding curvature tensor, $\phi=\phi_{1}+i \phi_{2}(i=\sqrt{-1})$ is a complex field on $\mathbb{R}^{3}$, called the Higgs field, $D_{\mu}=\frac{\partial}{\partial x^{\mu}}-i A_{\mu}$ is the gauge covariant derivative associated with $A_{\mu}, \varepsilon_{\mu \nu \rho}$ is the totally skewsymmetric tensor with $\varepsilon_{012}=1$, and finally $\kappa>0$ is the Chern-Simons coupling constant. Our metric on $\mathbb{R}^{3}$ is $\left(g_{\mu \nu}\right)=\operatorname{diag}(1,-1,-1)$. This model was suggested by HongKim-Pac[8] and Jackiw-Weinberg[10] to study vortex solutions of the Abelian Higgs model which carry both electric and magnetic charges(See [5] for a general survey of the model). This feature of the model is important in the

Key words and phrases. Chern-Simons theory, non-topological solutions.

This research supported partially by GARC-KOSEF, BSRI-MOE, KOSEF(K95070), SNU Research Fund and KIAS-M97003. 
physics of high critical temperature superconductivity. The Gauss equation (variational equation for $A_{0}$ ) of (1) is given by

$$
\kappa F_{12}=-2|\phi|^{2} A_{0} .
$$

Using this relation, and by integration by part the static energy corresponding to (1) can be written as $([8],[10])$

$$
\begin{aligned}
E & =\int_{\mathbb{R}^{2}}\left\{\frac{\kappa^{2}}{4} \frac{F_{12}^{2}}{|\phi|^{2}}+\sum_{j=1}^{2}\left|D_{j} \phi\right|^{2}+\frac{1}{\kappa^{2}}|\phi|^{2}\left(1-|\phi|^{2}\right)^{2}\right\} d x \\
& =\int_{\mathbb{R}^{2}}\left\{\left|\left(D_{1} \pm i D_{2}\right) \phi\right|^{2}+\left|\frac{\kappa F_{12}}{2 \phi} \pm \frac{1}{\kappa} \bar{\phi}\left(|\phi|^{2}-1\right)\right|^{2}\right\} d x \pm \int_{\mathbb{R}^{2}} F_{12} d x
\end{aligned}
$$

where $+(-)$ sign are chosen if the integral $\int_{\mathbb{R}^{2}} F_{12} d x$ has nonnegative(nonpositive) sign. Below we choose the upper sign. We have thus

$$
E \geq\left|\int_{\mathbb{R}^{2}} F_{12} d x\right|
$$

and the minimum of the energy is saturated if and only if $(\phi, A), A=\left(A_{1}, A_{2}\right)$ satisfies the self-duality equations, or the Bogomol'nyi equations:

$$
\begin{gathered}
\left(D_{1}+i D_{2}\right) \phi=0, \\
F_{12}+\frac{2}{\kappa^{2}}|\phi|^{2}\left(|\phi|^{2}-1\right)=0 .
\end{gathered}
$$

The system (4) -(5) is equipped with the following natural boundary conditions

$$
|\phi(x)| \rightarrow 1 \text { as }|x| \rightarrow \infty
$$

or

$$
|\phi(x)| \rightarrow 0 \text { as }|x| \rightarrow \infty
$$

in order to make the energy (3-a) finite. The solutions $(\phi, A)$ of (4)-(5) satisfying (6) are called topological solutions, while the solutions of (4)-(5) satisfying the boundary condition (7) are called nontopological solutions. Following Jaffe-Taubes [11], we can reduce system (4)-(5) with (6) or (7) 
to the more simplified form of partial differential equations as follows. We introduce new variable $(u, \theta)$ by

$$
\phi=e^{\frac{1}{2}(u+i \theta)}, \quad \theta=2 \sum_{j=1}^{N} \arg \left(z-z_{j}\right), \quad z=x_{1}+i x_{2} \in \mathbb{C}^{1}=\mathbb{R}^{2},
$$

where $z_{j}$, allowing multiplicities, $j=1,2, \ldots, N$ are the zeros, called the centres of the vorticities, of $\phi(z)$. Then, we can rewrite (4) -(5) with (6) or (7) as (Hereafter, we set $\kappa=2$ for simplicity.)

$$
\begin{gathered}
\Delta u=e^{u}\left(e^{u}-1\right)+4 \pi \sum_{j=1}^{N} \delta\left(z-z_{j}\right), \\
u(x) \rightarrow 0 \text { as }|x| \rightarrow \infty \quad \text { (topological boundary condition) }
\end{gathered}
$$

or

$$
u(x) \rightarrow-\infty \text { as }|x| \rightarrow \infty \quad \text { (non-topological boundary condition). }
$$

For topological boundary condition Wang[16] proved existence of general multivortex solutions, using the variational method similar to JaffeTaubes[11]. Later, Spruck-Yang [13] proved existence of topological solutions, using a more constructive iteration method, and generated even shapes of vortices by numerical simulations. (See [15] also for the study of (4)-(5) in a periodic bounded domain, and [3] for the study of topological solitons of the Chern-Simons model coupled with the Maxwell fields in a self-dual fashion.) The non-topological solutions, however, has not been well understood yet compared to the topological ones. In [14] Spruck-Yang proved existence of radially symmetric non-topological solutions which correspond to solutions of (4)-(5) with a single center(See [4] also for related studies of the radial solutions). In this paper we prove existence of general type of non-topological multivortex solutions. Our method of existence proof is quite constructive using an iteration scheme. Moreover, we establish precise decay estimates near infinity of our solutions. More specifically we prove the following theorem: 
Main Theorem. Let $\left\{z_{j}\right\}_{j=1}^{N} \subset \mathbb{C}^{1}$ be arbitrarily given. Then, there exists a solution $(\phi, A)$ to (4)-(5) (with $\kappa=2)$, (7) such that the function $\phi(z)$ has the zeros $\left\{z_{j}\right\}_{j=1}^{N}$ with possible multiplicities, and the pair $(\phi, A)$ make the energy functional (3-a) finite. Moreover, our constructed solutions satisfy:

(i) The decay estimates; there exists $\beta>0$ such that

$$
|\phi|^{2}+\left|F_{12}\right|+\left|D_{1} \phi\right|^{2}+\left|D_{2} \phi\right|^{2}=O\left(\frac{1}{|x|^{2 N+4+\beta}}\right) \text { as }|x| \rightarrow \infty
$$

(ii) Flux integral; there exists a positive constant $\gamma$, which depends on the solution, and can be fixed to be arbitrarily small, such that

$$
\Phi=\int_{\mathbb{R}^{2}} F_{12} d x=4 \pi(N+1)+\gamma
$$

Remark 1.

Since $|\phi|^{2}=e^{u}$, where $u$ is a solution of (9), we can deduce that if

$$
\lim _{|x| \rightarrow \infty} \frac{u(x)}{\ln |x|}=-B
$$

then necessarily we have $B>2 N+4$. It would be interesting to compare this result with the previously known one for the (See Theorem 2.1 of [14], and Theorem 3.2 of [4].) for the radially symmetric solutions of the following equation for voticities concentrated on the origin of $\mathbb{R}^{2}$.

$$
\Delta u=e^{u}\left(e^{u}-1\right)+4 \pi N \delta(z)
$$

equipped with the nontopological boundary condition (10). They proved existence of radially symmetric non-topological solution $u$ of (14) satisfying (13) with $B>2 N+4$. Thus our existence result is a "proper" generalization of the previous ones for the radially symmetric ones in [4] and [14].

Remark 2. Equation (12) implies that the integral $\int_{\mathbb{R}^{2}} F_{12} d x$, which corresponds to the minimum of the total static energy given by (3.a)-(3.b), is not "quantized" contrary to the case of topological solutions. This phenomena, which was discussed in the physics literature(See e.g. [5] and references therein) for radially symmetric solutions, is to the authors' knowledge rigorously verified first time in this paper for a nonradial solution. We also note that the estimate $\Phi>4(N+1) \pi$ was proved by Spruck-Yang[14] for 
any radially symmetric solution. The fact that $\gamma$ can be chosen arbitrarily small implies that their estimate is sharp for radially symmetric solution. For arbitrary nonradial solutions such estimate is currently not available.

Our idea of proof is the following: We formulate our problem of construction of nontopological solutions as one of finding roots of a functional equation defined in suitable function spaces. To find the roots we use the theorem on the convergence of a modified Newton's iteration scheme developed by Kantorovich [12]. Basic observation is that after suitable scale transformation our equation can be a perturbed one from a radially symmetric equation, and the iteration starting from a radial solution of the Liouville equation superposed with appropriate "small" nonradial function works well. The organization of the paper is the following. In Section 1 we introduce basic function spaces, and derive some properties of them useful in the following sections. In Section 2 we formulate our problem in terms of root finding of some mapping between function spaces introduced in Section 1. In Section 3 we prove various estimates on the mapping introduced in Section 2 in order to apply our iteration scheme. In Section 4 based on the estimates established in the previous section, we prove our main theorem, using the Newton-Kantorovich iteration scheme. We postpone all the proofs of the auxiliary lemmas to Appendix in order to help the readers to keep on the main stream of argument.

\section{Introduction of Function spaces}

In this section we define the class of functional spaces which are necessary in sequel and establish some properties of functions from these spaces. Let $\alpha \in(0,1)$ be given. We introduce the Hilbert spaces $X_{\alpha}$ and $Y_{\alpha}$ as follows

$$
X_{\alpha}=\left\{u(x) \in L_{l o c}^{2}\left(\mathbb{R}^{2}\right) \mid \int_{\mathbb{R}^{2}}\left(1+|x|^{2+\alpha}\right) u^{2} d x<\infty\right\},
$$

equipped with the inner product $(u, v)_{X_{\alpha}}=\int_{\mathbb{R}^{2}}\left(1+|x|^{2+\alpha}\right) u v d x$, and

$$
Y_{\alpha}=\left\{u \in W_{l o c}^{2,2}\left(\mathbb{R}^{2}\right) \mid\|\Delta u\|_{X_{\alpha}}^{2}+\left\|\frac{u}{1+|x|^{1+\frac{\alpha}{2}}}\right\|_{L^{2}\left(\mathbb{R}^{2}\right)}^{2}<\infty\right\}
$$

equipped with the inner product

$$
(u, v)_{Y_{\alpha}}=(\Delta u, \Delta v)_{X_{\alpha}}+\int_{\mathbb{R}^{2}} \frac{u v}{1+|x|^{2+\alpha}} d x .
$$


These spaces are equipped with the natural Banach space norms;

$$
\|u\|_{X_{\alpha}}=\sqrt{(u, u)_{X_{\alpha}}}, \quad\|u\|_{Y_{\alpha}}=\sqrt{(u, u)_{Y_{\alpha}}}
$$

respectively. Thanks to the inequality

$$
\int_{\mathbb{R}^{2}}|u| d x \leq\left(\int_{\mathbb{R}^{2}} \frac{1}{1+|x|^{2+\alpha}} d x\right)^{\frac{1}{2}}\left(\int_{\mathbb{R}^{2}}\left(1+|x|^{2+\alpha}\right) u^{2} d x\right)^{\frac{1}{2}}
$$

there is a continuous imbedding

$$
X_{\alpha} \hookrightarrow L^{1}\left(\mathbb{R}^{2}\right) \quad \forall \alpha \in(0,1) .
$$

Also, by the local regularity of the Laplace operator (See [7]) we have

$$
Y_{\alpha} \subset C_{l o c}^{0}\left(\mathbb{R}^{2}\right) \quad \forall \alpha \in(0,1) .
$$

We start from the following elementary proposition

Proposition 1.1. Let $\alpha \in(0,1)$ and $v \in Y_{\alpha}$ be a harmonic function. Then $v \equiv$ const.

Proof. In the polar coordinates the Fourier expansion in $\theta$ yields

$$
v(x)=\sum_{k=0}^{\infty} v_{k}(r) e^{i k \theta}
$$

with the rapidly decreasing coefficients, $v_{k}(r)$ in $k$ for each fixed $r \geq 0$. Since $v(x)$ is the harmonic function, each $v_{k}(r)$ satisfies the ordinary differential equation:

$$
\frac{d^{2} v_{k}}{d r^{2}}+\frac{1}{r} \frac{d v_{k}}{d r}-\frac{k^{2}}{r^{2}} v_{k}=0 \quad \forall k \in \mathbb{Z}_{+} \cup\{0\} .
$$

The general solution of these ordinary differential equations is well known:

$$
v_{k}(r)=M_{1, k} r^{k}+\frac{M_{2, k}}{r^{k}} \quad \text { for } k \in \mathbb{Z}_{+}, \quad v_{0}(r)=M_{1,0}+M_{2,0} \ln r,
$$

where $M_{1, k}, M_{2, k}$ are the constants. Noting $\int_{-\pi}^{\pi} e^{i k \theta} d \theta$ equals 0 for $k \neq 0$ and $2 \pi$ for $k=0$, we deduce

$$
v_{k} \in X_{\alpha} \quad \forall k \in \mathbb{Z}_{+} \cup\{0\} .
$$

Hence $M_{1, k}=0$ for all $k \in \mathbb{Z}_{+}$. Then taking into account that $v$ is smooth function in neighborhood of zero we obtain $M_{2, k}=0$ for all $k \in \mathbb{Z}_{+} \cup\{0\}$.

Denote $\ln ^{+}|x|=\max \{0, \ln |x|\}$ below. The next lemma provides the pointwise estimate for an arbitrary function from $Y_{\alpha}$. In particular it imply that on infinity functions from this space have at most logarithmic growth. 
Lemma 1.1. Let $\alpha \in(0,1)$, then there exists $C_{1}>0$ such that for all $v \in Y_{\alpha}$

$$
|v(x)| \leq C_{1}\|v\|_{Y_{\alpha}}\left(\ln ^{+}|x|+1\right) \quad \forall x \in \mathbb{R}^{2} .
$$

Proof. For given $v \in Y_{\alpha}$, we set $\Delta v=g$. By definition of the space $Y_{\alpha}$ the function $g \in X_{\alpha}$. We consider the function

$$
\tilde{v}(x)=\frac{1}{2 \pi} \int_{\mathbb{R}^{2}} \ln |x-\tau| g(\tau) d \tau .
$$

It is well-known that $\Delta \tilde{v}=g$ in $\mathbb{R}^{2}$. Using the Cauchy-Bynakovskii inequality we obtain

$$
\begin{aligned}
|\tilde{v}(x)| & \leq \frac{1}{2 \pi}\left|\int_{\mathbb{R}^{2}} \ln \right| x-\tau|g(\tau) d \tau| \leq \frac{1}{2 \pi} \int_{\mathbb{R}^{2}}|\ln | x-\tau \||g(\tau)| d \tau \\
& \leq \frac{1}{2 \pi}\left(\int_{\mathbb{R}^{2}}\left(1+|\tau|^{2+\alpha}\right)|g(\tau)|^{2} d \tau\right)^{\frac{1}{2}}\left(\int_{\mathbb{R}^{2}} \frac{\ln ^{2}|x-\tau|}{1+|\tau|^{2+\alpha}} d \tau\right)^{\frac{1}{2}} \\
& \leq \frac{1}{2 \pi}\|v\|_{Y_{\alpha}}\left(\int_{\mathbb{R}^{2}} \frac{\ln ^{2}|\tau|}{1+|x-\tau|^{2+\alpha}} d \tau\right)^{\frac{1}{2}} .
\end{aligned}
$$

Let us estimate the last integral in (1.4)

$$
\begin{aligned}
I^{2} & =\int_{\mathbb{R}^{2}} \frac{\ln ^{2}|\tau|}{1+|x-\tau|^{2+\alpha}} d \tau \\
& =\int_{|\tau| \leq 1} \frac{\ln ^{2}|\tau|}{1+|x-\tau|^{2+\alpha}} d \tau+\int_{|\tau| \geq 1} \frac{\ln ^{2}|\tau|}{1+|x-\tau|^{2+\alpha}} d \tau \\
& =A_{1}+A_{2} .
\end{aligned}
$$

Obviously,

$$
A_{1}=\int_{|\tau| \leq 1} \frac{\ln ^{2}|\tau|}{1+|x-\tau|^{2+\alpha}} d \tau \leq \int_{|\tau| \leq 1} \ln ^{2}|\tau| d \tau \leq C .
$$

Note that for $|\tau| \geq 2|x|$ we have

$$
|\tau-x| \geq|\tau|-|x|=\frac{1}{2}|\tau|+\frac{1}{2}|\tau|-|x| \geq \frac{1}{2}|\tau| .
$$

Thus, 


$$
\begin{aligned}
\int_{|\tau| \geq 1} \frac{\ln ^{2}|\tau|}{1+|x-\tau|^{2+\alpha}} d \tau=\int_{\{|\tau| \geq 1\} \cap\{|\tau| \geq 2|x|\}} \frac{\ln ^{2}|\tau|}{1+|x-\tau|^{2+\alpha}} d \tau \\
\quad+\int_{\{|\tau| \geq 1\} \cap\{|\tau|<2|x|\}} \frac{\ln ^{2}|\tau|}{1+|x-\tau|^{2+\alpha}} d \tau \leq C\left(1+\left(\ln ^{+} 2|x|\right)^{2}\right)
\end{aligned}
$$

where the constants $C$ depends on $\alpha$ only. Then inequalities (1.4)(1.6) imply

$$
|\tilde{v}(x)| \leq C\|v\|_{Y_{\alpha}}\left(\ln ^{+}|x|+1\right) \quad \forall x \in \mathbb{R}^{2} .
$$

Hence $\tilde{v} \in Y_{\alpha}$ and by Proposition 1.1 there exists $k \in \mathbb{R}$ such that $\tilde{v}(x)=$ $v(x)+k$. Thus the pointwise estimate (1.7) yields

$$
\|v+k\|_{Y_{\alpha}} \leq C\|v\|_{Y_{\alpha}} .
$$

Hence

$$
|k| \leq C\|k\|_{Y_{\alpha}} \leq C\left(\|v+k\|_{Y_{\alpha}}+\|v\|_{Y_{\alpha}}\right) \leq C\|v\|_{Y_{\alpha}} .
$$

Then by (1.7) and this estimate we obtain (1.3).

\section{Functional formulation of the PRoblem}

The aim of this section is two-fold. First we wish to transform equation (9) to more convenient form, and second we would like to outline the strategy of the proof of the main theorem.

As the first step in transforming equation (9) we get rid of delta-functions in its right hand side, using solutions for the Liouville equation.

Throughout this paper we denote $z=x_{1}+i x_{2}, a=a_{1}+i a_{2} \in \mathbb{C}^{1}=\mathbb{R}^{2}$. Let us define

$$
f(z)=(N+1) \prod_{k=1}^{N}\left(z-z_{k}\right),
$$

and

$$
F(z)=\int_{0}^{z} f(\xi) d \xi .
$$

Let us introduce a functions $\rho_{\varepsilon, a}(z), \rho(r)$ by

$$
\rho_{\varepsilon, a}(z)=\frac{8 \varepsilon^{2 N+2}|f(z)|^{2}}{\left(1+\varepsilon^{2 N+2}\left|F(z)+\frac{a}{\varepsilon^{N+3}}\right|^{2}\right)^{2}}, \quad \rho(r)=\frac{8(N+1)^{2} r^{2 N}}{\left(1+r^{2 N+2}\right)^{2}} .
$$


We note that for any $\varepsilon>0$ and $a \in \mathbb{C}^{1}, \ln \rho_{\varepsilon, a}(z)=\Theta(z)$ is a solution of the Liouville equation

$$
\Delta \Theta+e^{\Theta}=4 \pi \sum_{j=1}^{N} \delta\left(z-z_{j}\right)
$$

in $\mathbb{R}^{2}$.

Defining $v(z)=u(z)-\ln \rho_{\varepsilon, a}(z)$, we obtain from $(9)$

$$
\Delta v+\rho_{\varepsilon, a} e^{v}-\rho_{\varepsilon, a}^{2} e^{2 v}-\rho_{\varepsilon, a}=0 .
$$

Then, making change of variables $z \rightarrow \frac{z}{\varepsilon}$ and denoting $\tilde{v}(z)=v\left(\frac{z}{\varepsilon}\right)$, we have

$$
\begin{array}{r}
\Delta \tilde{v}+\frac{8 \varepsilon^{2 N}\left|f\left(\frac{z}{\varepsilon}\right)\right|^{2} e^{\tilde{v}}}{\left(1+\varepsilon^{2 N+2}\left|F\left(\frac{z}{\varepsilon}\right)+\frac{a}{\varepsilon^{N+3}}\right|^{2}\right)^{2}}-\frac{64 \varepsilon^{4 N+2}\left|f\left(\frac{z}{\varepsilon}\right)\right|^{4} e^{2 \tilde{v}}}{\left(1+\varepsilon^{2 N+2}\left|F\left(\frac{z}{\varepsilon}\right)+\frac{a}{\varepsilon^{N+3}}\right|^{2}\right)^{4}} \\
-\frac{8 \varepsilon^{2 N}\left|f\left(\frac{z}{\varepsilon}\right)\right|^{2}}{\left(1+\varepsilon^{2 N+2}\left|F\left(\frac{z}{\varepsilon}\right)+\frac{a}{\varepsilon^{N+3}}\right|^{2}\right)^{2}}=0 .
\end{array}
$$

We denote

$$
g_{\varepsilon}(z, a)=\frac{8 \varepsilon^{2 N}\left|f\left(\frac{z}{\varepsilon}\right)\right|^{2}}{\left(1+\left|\varepsilon^{N+1} F\left(\frac{z}{\varepsilon}\right)+\frac{a}{\varepsilon^{2}}\right|^{2}\right)^{2}} .
$$

Then, we can write $(2.3)$ as

$$
\Delta \tilde{v}+g_{\varepsilon} e^{\tilde{v}}-\varepsilon^{2} g_{\varepsilon}^{2} e^{2 \tilde{v}}-g_{\varepsilon}=0
$$

To transform (2.5) further we construct a solution to the ordinary differential equation

$$
L_{1} w=\frac{d^{2} w}{d r^{2}}+\frac{1}{r} \frac{d w}{d r}+\rho w=f \quad \text { in } \mathbb{R}_{+} .
$$

We first observe that that the function $\varphi_{0}(r)$ defined by

$$
\varphi_{0}(r)=\frac{1-r^{2 N+2}}{1+r^{2 N+2}}
$$

solves (2.6) with $f=0$.(For rational derivation of this fact please see the proof of Lemma 2.4.) From this we establish the following two lemmas, the proofs of which are in Appendix. 
Lemma 2.1. Given $f=f(r)$ with $r f(r) \in L^{1}\left(\mathbb{R}_{+}\right), f(r) \in C^{1}\left(\mathbb{R}_{+}\right)$, the ordinary differential equation (2.6) has an explicit solution

$$
w(r)=\varphi_{0}(r)\left\{\int_{0}^{r} \frac{\phi_{f}(s)-\phi_{f}(1)}{(1-s)^{2}} d s+\frac{\phi_{f}(1) r}{1-r}\right\}
$$

with

$$
\phi_{f}(r)=\left(\frac{1+r^{2 N+2}}{1-r^{2 N+2}}\right)^{2} \frac{(1-r)^{2}}{r} \int_{0}^{r} \varphi_{0}(t) t f(t) d t
$$

where $\phi_{f}(1)$ and $w(1)$ are defined as limits of $\phi_{f}(r)$ and $w(r)$ as $r \rightarrow 1$.

Lemma 2.2. Let $w_{0}(r)$ be a solution of the equation

$$
L_{1} w_{0}-\rho^{2}=0 \quad \text { in } \mathbb{R}_{+},
$$

obtained by substituting $f=\rho^{2}$ in the solution formula (2.7)-(2.8). Then, the pointwise estimates hold true

$$
\sup _{r>0} e^{w_{0}(r)}<\infty, \quad\left|w_{0}(r)\right| \leq C\left(\ln ^{+} r+1\right) \quad \forall r>0,
$$

and the asymptotic formula

$$
w_{0}(r)=-\tilde{C} \ln r+o(\ln r) \quad \text { as } r \rightarrow \infty,
$$

where $C$ and $\tilde{C}$ are positive constants independent of $r$.

Let us introduce the mapping $P_{\varepsilon}(\cdot, \cdot): Y_{\alpha} \times \mathbb{R}^{2} \mapsto X_{\alpha}$ given by

$$
P_{\varepsilon}(u, a)=\Delta u+g_{\varepsilon}(z, a) e^{u+\varepsilon^{2} w_{0}}-\varepsilon^{2} g_{\varepsilon}^{2}(z, a) e^{2\left(u+\varepsilon^{2} w_{0}\right)}-g_{\varepsilon}(z, a)+\varepsilon^{2} \Delta w_{0},
$$

where $w_{0}(x)=w_{0}(|x|)$ is the function defined by Lemma 2.2. Then, the problem of solving equation (9) is reduced to that of finding a root, $\left(u_{\varepsilon}^{*}, a_{\varepsilon}^{*}\right) \in$ $Y_{\alpha} \times \mathbb{R}^{2}$ of the functional equation

$$
P_{\varepsilon}(u, a)=0 \text {. }
$$

The transformation of equation (9) is finished.

Finally we note that once a solution of $(2.12),\left(u_{\varepsilon}^{*}, a_{\varepsilon}^{*}\right)$ is found, then our solution $u$ of the equation (9) is recovered by the formula

$$
u(x)=\ln \rho_{\varepsilon, a_{\varepsilon}^{*}}(x)+u_{\varepsilon}^{*}(\varepsilon x)+\varepsilon^{2} w_{0}(\varepsilon x) .
$$

Of course, after that one should check if this solution really satisfies the nontopological boundary conditions.

The proof of our main theorem is based on the following theorem due to Kantorovich [12,p. 532]: 
Theorem 2.1. Let $B_{1}$ and $B_{2}$ be Banach spaces, and $\Omega \subset B_{1}$ be an arbitrary domain, $P: \Omega \rightarrow B_{2}$ be a given mapping which has a continuous second derivative in $\Omega_{0}$, where $\Omega_{0}=\left\{v \in \Omega \mid\left\|v-v_{0}\right\|_{B_{1}} \leq \tilde{r}\right\}$. Suppose, in addition, that

(i) $\Gamma_{0}=\left[P^{\prime}\left(v_{0}\right)\right]^{-1}$ exists and continuous linear operator;

(ii) $\left\|\Gamma_{0}\left(P\left(v_{0}\right)\right)\right\| \leq \eta$;

(iii) $\left\|\Gamma_{0} P^{\prime \prime}(v)\right\| \leq K \quad \forall v \in \Omega_{0}$;

Then, provided

$$
h=K \eta \leq \frac{1}{2}, \quad \text { and } \tilde{r} \geq r_{0}=\frac{1-\sqrt{1-2 h}}{h} \eta
$$

The functional equation

$$
P(v)=0 \quad \text { in } \Omega
$$

has a solution $v^{*}$ such that

$$
\left\|v^{*}-v_{0}\right\|_{B_{1}} \leq r_{0}
$$

Later, we apply the Newton-Kantorovich iteration method to solve (2.12), starting from the origin of some subspace of the space $Y_{\alpha} \times \mathbb{R}^{2}$. For this we need to estimate the distance in $X_{\alpha}$ from origin to

$$
P_{\varepsilon}(0,0)=g_{\varepsilon}(z, 0) e^{\varepsilon^{2} w_{0}}-\varepsilon^{2} g_{\varepsilon}^{2}(z, 0) e^{2 \varepsilon^{2} w_{0}}-g_{\varepsilon}(z, 0)+\varepsilon^{2} \Delta w_{0}
$$

and prove the invertibility of the first derivative of $P_{\varepsilon}$ at the origin,

$$
\begin{aligned}
P_{\varepsilon}^{\prime}(0,0)[v, b]= & \Delta v+g_{\varepsilon}(z, 0) e^{\varepsilon^{2} w_{0}} v-2 \varepsilon^{2} g_{\varepsilon}^{2}(z, 0) e^{2 \varepsilon^{2} w_{0}} v \\
+ & \sum_{j=1}^{2} \frac{\partial g_{\varepsilon}(z, 0)}{\partial a_{j}} e^{\varepsilon^{2} w_{0}} b_{j}-2 \varepsilon^{2} \sum_{j=1}^{2} \frac{\partial g_{\varepsilon}(z, 0)}{\partial a_{j}} g_{\varepsilon}(z, 0) e^{2 \varepsilon^{2} w_{0}} b_{j} \\
& -\sum_{j=1}^{2} \frac{\partial g_{\varepsilon}(z, 0)}{\partial a_{j}} b_{j}
\end{aligned}
$$


and finally we need to estimate the norm of the second derivative,

$$
\begin{aligned}
P_{\varepsilon}^{\prime \prime}(u, a)[v, b ; \tilde{v}, \tilde{b}]= & g_{\varepsilon}(z, a) e^{u+\varepsilon^{2} w_{0}} v \tilde{v}+\sum_{j=1}^{2} \frac{\partial g_{\varepsilon}(z, a)}{\partial a_{j}} e^{u+\varepsilon^{2} w_{0}} v \tilde{b}_{j} \\
& +\sum_{j=1}^{2} \frac{\partial g_{\varepsilon}(z, a)}{\partial a_{j}} e^{u+\varepsilon^{2} w_{0}} b_{j} \tilde{v}+\sum_{j, k=1}^{2} \frac{\partial^{2} g_{\varepsilon}(z, a)}{\partial a_{j} \partial a_{k}} e^{u+\varepsilon^{2} w_{0}} b_{j} \tilde{b}_{k} \\
& -4 \varepsilon^{2} \sum_{j=1}^{2} \frac{\partial g_{\varepsilon}(z, a)}{\partial a_{j}} g_{\varepsilon}(z, a) e^{2 u+2 \varepsilon^{2} w_{0}} b_{j} \tilde{v} \\
& -2 \varepsilon^{2} \sum_{j, k=1}^{2}\left(\frac{\partial^{2} g_{\varepsilon}(z, a)}{\partial a_{j} \partial a_{k}} g_{\varepsilon}(z, a)+\frac{\partial g_{\varepsilon}(z, a)}{\partial a_{j}} \frac{\partial g_{\varepsilon}(z, a)}{\partial a_{k}}\right) b_{j} \tilde{b}_{k} e^{2 u+2 \varepsilon^{2}} \\
& -4 \varepsilon^{2} g_{\varepsilon}^{2}(z, a) e^{2 u+2 \varepsilon^{2} w_{0}} v \tilde{v} \\
& -4 \varepsilon^{2} \sum_{j=1}^{2} \frac{\partial g_{\varepsilon}(z, a)}{\partial a_{j}} g_{\varepsilon}(z, a) e^{2 u+2 \varepsilon^{2} w_{0}} v \tilde{b}_{j} \\
& -\sum_{j, k=1}^{2} \frac{\partial^{2} g_{\varepsilon}(z, a)}{\partial a_{j} \partial a_{k}} b_{j} \tilde{b}_{k}
\end{aligned}
$$

at the origin.

The most difficult part is to prove the invertivility of the operator $\left[P_{\varepsilon}^{\prime}(0,0)\right]$. To solve this problem let us introduce an operator $A: Y_{\alpha} \times \mathbb{R}^{2} \rightarrow X_{\alpha}$ defined by

$$
A\left(u, a_{1}, a_{2}\right)=L u+M a,
$$

where the operator $L: Y_{\alpha} \mapsto X_{\alpha}$ is defined by

$$
L u=\Delta u+\frac{8(N+1)^{2} r^{2 N} u}{\left(1+r^{2 N+2}\right)^{2}}, \quad r=|x|,
$$

and the operator $M: \mathbb{R}^{2} \mapsto X_{\alpha}$ is defined by

$$
M a=-4\left(\rho w_{0}-2 \rho^{2}\right) \varphi_{+} a_{1}-4\left(\rho w_{0}-2 \rho^{2}\right) \varphi_{-} a_{2} \quad \forall a=\left(a_{1}, a_{2}\right) \in \mathbb{R}^{2}
$$

with the functions $\varphi_{+}(r, \theta), \quad \varphi_{-}(r, \theta) \in Y_{\alpha}$ given by formulas

$$
\varphi_{+}(r, \theta)=\frac{r^{N+1} \cos (N+1) \theta}{1+r^{2 N+2}}, \quad \varphi_{-}(r, \theta)=\frac{r^{N+1} \sin (N+1) \theta}{1+r^{2 N+2}} .
$$

Later, we shall prove that the operator $P_{\varepsilon}^{\prime}(0,0)$ is a perturbation of the operator $A$ in the norm $\mathcal{L}\left(Y_{\alpha} \times \mathbb{R}^{2}, X_{\alpha}\right)$. Now let us prove the following property of the operator $A$. 
Theorem 2.2. The operator $A$ defined by (2.17)-(2.20) belongs to $\mathcal{L}\left(Y_{\alpha} \times\right.$ $\left.\mathbb{R}^{2}, X_{\alpha}\right)$ and

$$
\operatorname{Im} A=X_{\alpha} .
$$

To prove this proposition first we establish facts on image and kernel of the operator $L$.

We start from the following:

Proposition 2.1. Let $\alpha \in\left(0, \frac{1}{2}\right)$, then the image of $L, \operatorname{Im} L$ is closed in $X_{\alpha}$.

The proof of Proposition 2.1 follows immediately from the Lemma below.

Lemma 2.3. Suppose that $X, Y$ are Hilbert spaces, a bounded linear operator $B: X \mapsto Y$ is onto, and $K: X \mapsto Y$ is a linear compact operator. Then, Im $(B+K)$ is closed in $Y$.

Proof. See the proof of Lemma 5.1 of [6], page 413.

Proof of Proposition 2.1. We apply Lemma 2.3 for operators $B=\Delta, K=\rho$. Now, it is easy to check $K: Y_{\alpha} \mapsto X_{\alpha}$ is a compact operator, by decomposing $\mathbb{R}^{2}$ into a bounded and an exterior domains, and using the RellichKondrachev compactness lemma for the bounded domain. We omit details of this part. Here, we only check $\Delta: Y_{\alpha} \rightarrow X_{\alpha}$ is onto. Given $f \in X_{\alpha}$, let us consider a solution $u$ of the equation

$$
\Delta u=f \quad \text { in } \mathbb{R}^{2}
$$

represented by

$$
u(x)=\frac{1}{2 \pi} \int_{\mathbb{R}^{2}} \ln |x-y| f(y) d y .
$$

We will show $u \in Y_{\alpha}$. First $\|\Delta u\|_{X_{\alpha}}<\infty$ is immediate. Next, we claim

$$
\int_{\mathbb{R}^{2}} \frac{u^{2}(x)}{1+|x|^{2+\alpha}} d x<\infty .
$$

Indeed, we have

$$
\begin{aligned}
|u(x)| & \leq \frac{1}{2 \pi} \int_{\mathbb{R}^{2}} \frac{|\ln | x-y||}{\left(1+|y|^{2+\alpha}\right)^{\frac{1}{2}}}\left(1+|y|^{2+\alpha}\right)^{\frac{1}{2}}|f(y)| d y \\
& \leq \frac{1}{2 \pi}\left(\int_{\mathbb{R}^{2}} \frac{\ln ^{2}|x-y|}{1+|y|^{2+\alpha}} d y\right)^{\frac{1}{2}}\|f\|_{X_{\alpha}} \\
& \leq C\|f\|_{X_{\alpha}}\left(\ln ^{+}|x|+1\right),
\end{aligned}
$$


where we used previous estimate for $I$ in the proof of Lemma 1.1(See (1.5)(1.6)). Thus (2.21) follows, and $u \in Y_{\alpha}$.

Now we can compute the kernel of the operator $L$.

Lemma 2.4. Let $\alpha \in\left(0, \frac{1}{2}\right)$, and $L: Y_{\alpha} \mapsto X_{\alpha}$ be the differential operator defined by (2.18). Then,

$$
\operatorname{ker} L=\operatorname{Span}\left\{\varphi_{0}, \varphi_{+}, \varphi_{-}\right\}
$$

where $\varphi_{0}, \varphi_{ \pm}$are introduced in (2.7) and (2.20) respectively.

Proof. As is well-known, for any $N, k \in \mathbb{Z}_{+} \cup\{0\}$ and $a=a_{1}+i a_{2} \in \mathbb{C}^{1}$ the function

$$
\Psi(a, z)=\ln \frac{8\left|(N+1) z^{N}+a(k+N+1) z^{N+k}\right|^{2}}{\left(1+\left|z^{N+1}+a z^{N+k+1}\right|^{2}\right)^{2}} .
$$

satisfies the Liouville equation

$$
\Delta \Psi+e^{\Psi}=0
$$

except at the zeros of the polynomial $p(z)=(N+1) z^{N}+a(k+N+1) z^{N+k}$ in $\mathbb{C}^{1}$. Taking derivative of $(2.22)$ with respect to $a_{1}$ and $a_{2}$ at the point $a=0$, we find that for each $k \in \mathbb{Z}_{+} \cup\{0\}$ the functions

$$
\varphi_{+, k}=\left.\frac{\partial \Psi(a, z)}{\partial a_{1}}\right|_{a=0}=\phi_{k}(r) \cos k \theta, \quad \varphi_{-, k}=\phi_{k}(r) \sin k \theta
$$

with

$$
\phi_{k}(r)=\frac{(k+N+1)+(k-N-1) r^{2 N+2}}{(N+1)\left(1+r^{2 N+2}\right)} r^{k}
$$

satisfy the equation

$$
L \varphi_{ \pm, k}=0 \quad \text { in } \mathbb{R}^{2}
$$

and function $\phi_{k}$ satisfy to the ordinary differential equation

$$
\frac{\partial^{2} \phi_{k}}{\partial r^{2}}+\frac{1}{r} \frac{\partial \phi_{k}}{\partial r}-\frac{k^{2} \phi_{k}}{r^{2}}+\rho \phi_{k}=0 \quad \forall r>0 .
$$

On the other hand, as can be checked by direct computations, new functions $\tilde{\phi}_{k}(r)=\phi_{k}(1 / r)$ are also solutions to the equation (2.23). Obviously the pair $\phi_{k}, \tilde{\phi}_{k}$ is the fundamental set of solutions to ordinary differential 
equation (2.23). If $k \notin\{0, N+1\}$, then these solutions blow up like $r^{k}$ near infinity, or like $r^{-k}$ near 0 , and do not belong to the space $Y_{\alpha}$.

Now let $v(x) \in \operatorname{ker} L \cap W_{l o c}^{2,2}\left(\mathbb{R}^{2}\right)$. Using well-known regularity results of elliptic operators we obtain that $v(x) \in C_{l o c}^{2}\left(\mathbb{R}^{2}\right)$. In the polar coordinates the Fourier expansion yields

$$
v(x)=\sum_{k=0}^{\infty} v_{k}(r) e^{i k \theta}
$$

Obviously the function $v_{k}(r)$ satisfies $(2.23)$ for all $k \in \mathbb{Z}_{+} \cup\{0\}$. Thus $v_{k}(r)$ is the linear combination of the functions $\phi_{k}(r), \tilde{\phi}_{k}(r)$

$$
v_{k}(r)=c_{k, 1} \phi_{k}(r)+c_{k, 2} \tilde{\phi}_{k}(r)
$$

Since $v$ is the smooth function $c_{k, 2}=0$. On the other hand $v(x) \in Y_{\alpha}$, thus $c_{k, 1}=0$ for all $k \in \mathbb{Z}_{+} \backslash\{N+1\}$. This proves the lemma.

The image of the operator $L$ is completely described by the following proposition.

Proposition 2.2. Let $\alpha \in\left(0, \frac{1}{2}\right)$, then for the image of the operator $L$ defined by (2.1) and (2.18) we have

$$
\operatorname{Im} L=\left\{f \in X_{\alpha} \quad \mid \quad \int_{\mathbb{R}^{2}} f \varphi_{ \pm} d x=0\right\}
$$

Proof. Since the image $\operatorname{Im} L$ is closed in $X_{\alpha}$ by Proposition 2.1, we can decompose $X_{\alpha}$ as follows:

$$
X_{\alpha}=\operatorname{Im} L \oplus(\operatorname{Im} L)^{\perp}
$$

Let $\xi \in(\operatorname{Im} L)^{\perp}$, then

$$
(L u, \xi)_{X_{\alpha}}=0 \quad \forall u \in Y_{\alpha}
$$

Thus, denoting $\left(1+|x|^{2+\alpha}\right) \xi(x)=\psi(x)$, we have

$$
(L u, \psi)_{L^{2}\left(\mathbb{R}^{2}\right)}=0 \quad \forall u \in Y_{\alpha} .
$$


Since $C_{0}^{\infty}\left(\mathbb{R}^{2}\right) \subset Y_{\alpha}$, this implies immediately that

$$
L \psi=0 \quad \text { in } \mathbb{R}^{2}
$$

by integration by parts and standard density argument.

Since $\xi \in X_{\alpha}$, we have $\int_{\mathbb{R}^{2}} \frac{|\psi|^{2}}{1+|x|^{2+\alpha}} d x<\infty$, and thus by (2.24)

$$
\psi \in \operatorname{ker} L \cap Y_{\alpha}
$$

By Lemma 2.4 the the function $\psi$ is a linear combination of $\varphi_{0}, \varphi_{ \pm}$,

$$
\psi=C_{0} \varphi_{0}+C_{+} \varphi_{+}+C_{-} \varphi_{-},
$$

where $C_{0}, C_{ \pm}$are constants. Let us consider a function $f(r)=\lambda(r) \varphi_{0}(r)$, where $\lambda(r)$ is a smooth cut-off function which is nonnegative, and whose non empty support is in $\left(\frac{1}{2}, 1\right)$. Let $w$ be the solution of $L_{1} w=f$ given by (2.8). Now suppose $C_{0}$ is not equal to 0 , then this leads us to the contradiction,

$$
\int_{\frac{1}{2}}^{1} C_{0} \lambda(r) \varphi_{0}^{2}(r) r d r=\int_{0}^{\infty} L_{1} w(r) C_{0} \varphi_{0}(r) r d r=C_{0} \int_{\mathbb{R}^{2}} L w \varphi_{0} d x=0 .
$$

Let $m(r) \in C^{\infty}\left(\mathbb{R}_{+}^{1}\right), m(r)=1$ for $r \in[0,1]$ and $m(r)=0$ for $r>2$. Denote $m_{\varepsilon}(x)=m(\varepsilon x)$. Obviously

$$
\varphi_{ \pm} m_{\varepsilon} \rightarrow \varphi_{ \pm} \quad \text { in } X_{\delta} \quad \text { as } \varepsilon \rightarrow+0,
$$

for all $\delta<-2$. Thus, using (1.3), we have

$$
\begin{aligned}
& \left(L u, \varphi_{ \pm}\right)_{L^{2}\left(\mathbb{R}^{2}\right)}=\lim _{\varepsilon \rightarrow+0}\left(L u, \varphi_{ \pm} m_{\varepsilon}\right)_{L^{2}\left(\mathbb{R}^{2}\right)} \\
= & \left.\lim _{\varepsilon \rightarrow+0} \int_{\mathbb{R}^{2}}\left\{\varepsilon^{2}(\Delta m)(\varepsilon r) \varphi_{ \pm} u+2 \varepsilon\left((\nabla m)(\varepsilon r), \nabla \varphi_{ \pm}\right) u\right)\right\} d x=0 \quad \forall u \in Y_{\alpha} .
\end{aligned}
$$

This completes the proof.

In (2.17)-(2.20) the operator $A$ was introduced by $A(u, a)=L u+M a$. Since variables $u$ and $a$ are independent, and $\operatorname{Im} L$ is the linear space of codimension we can prove Theorem 2.2 by showing that the space $(\operatorname{ImL} L)^{\perp}$ does not contain any vector orthogonal to $\operatorname{ImM}$. This goal will be achieved with the help of the following auxiliary Lemma, whose proof is given in Appendix. 
Lemma 2.5. The following inequality holds true:

$$
\int_{\mathbb{R}^{2}}\left(\rho w_{0}-2 \rho^{2}\right) \varphi_{ \pm}^{2} d x<0
$$

Now we can prove Theorem 2.2.

Proof of Theorem 2.2. Given $f \in X_{\alpha}$, we want to show that there exists $u \in Y_{\alpha}, a_{1}, a_{2} \in \mathbb{R}$ such that $A\left(u, a_{1}, a_{2}\right)=f$. Let us define

$$
C_{ \pm}=\int_{\mathbb{R}^{2}} f \varphi_{ \pm} d x, \quad \tilde{C}_{ \pm}=4 \int_{\mathbb{R}^{2}}\left(\rho w_{0}-2 \rho^{2}\right) \varphi_{ \pm}^{2} d x
$$

We note that $\tilde{C}_{ \pm} \neq 0$ due to Lemma 2.5. Thus, the function, $\tilde{f}$ introduced below, is well defined.

$$
\tilde{f}=f-\frac{4 C_{+}}{\tilde{C}_{+}}\left(\rho w_{0}-2 \rho^{2}\right) \varphi_{+}-\frac{4 C_{-}}{\tilde{C}_{-}}\left(\rho w_{0}-2 \rho^{2}\right) \varphi_{-} .
$$

Then, from $\int_{0}^{2 \pi} \sin (N+1) \theta \cos (N+1) \theta d \theta=0$ we obtain

$$
\int_{\mathbb{R}^{2}} \tilde{f} \varphi_{ \pm} d x=0
$$

Hence, by Proposition 2.2 there exists $u \in Y_{\alpha}$ such that $\Delta u+\rho u=\tilde{f}$, and we have

$$
A\left(u,-\frac{C_{+}}{\tilde{C}_{+}},-\frac{C_{-}}{\tilde{C}_{-}}\right)=f .
$$

This completes the proof of the proposition.

\section{Preliminary Estimates}

Our aim in this section is the proof of invertibility of $P_{\varepsilon}^{\prime}(0,0)$, and appropriate norm estimates for $P_{\varepsilon}^{\prime}(0,0)^{-1}, P_{\varepsilon}(0,0)$, and $P_{\varepsilon}^{\prime \prime}(0,0)$ respectively. For those purposes we need the following lemmas. 
Lemma 3.1. Suppose $g_{\varepsilon}(z, a)$ is the function defined in (2.4). Then, there exists $R_{0}>1$ and $\varepsilon \in(0,1)$ such that $|z| \geq R_{0}$ and $|a|<\varepsilon^{2}, \varepsilon \in\left(0, \varepsilon_{0}\right)$ imply

$$
\begin{gathered}
\left|g_{\varepsilon}(z, a)\right| \leq \frac{C|z|^{2 N}}{\left(1+|z|^{2 N+2}\right)^{2}}, \\
\sum_{j=1}^{2}\left|\frac{\partial g_{\varepsilon}(z, a)}{\partial a_{j}}\right| \leq \frac{C|z|^{2 N}}{\varepsilon^{2}\left(1+|z|^{2 N+2}\right)^{2}}, \\
\sum_{j, k=1}^{2}\left|\frac{\partial^{2} g_{\varepsilon}(z, a)}{\partial a_{j} \partial a_{k}}\right| \leq \frac{C|z|^{2 N}}{\varepsilon^{4}\left(1+|z|^{2 N+2}\right)^{2}},
\end{gathered}
$$

where $C$ is independent of $\varepsilon, z$.

The proof of this Lemma is given in Appendix.

Lemma 3.2. For all $\varepsilon_{0} \in(0,1)$ there exists $C=C\left(\varepsilon_{0}\right)$ such that

$$
e^{\varepsilon^{2} w_{0}}=1+\varepsilon^{2} w_{0}+R(r) \quad \forall \varepsilon \in\left(0, \varepsilon_{0}\right), \quad r \in \mathbb{R}_{+},
$$

with

$$
|R(r)| \leq C \varepsilon^{4}\left(\ln ^{+} r+1\right)^{2} \quad \forall r \in \mathbb{R}_{+} .
$$

Proof. By Taylor's formula we have

$$
e^{\varepsilon^{2} w_{0}(r)}=1+\varepsilon^{2} w_{0}(r)+R(r) \quad \forall r \in \mathbb{R}_{+}, \quad \varepsilon \in(0,1),
$$

where

$$
\begin{aligned}
|R(r)| & <\frac{1}{2} \sup _{\tau \in(0,1)} e^{\tau \varepsilon^{2} w_{0}(r)}\left|\varepsilon^{2} w_{0}(r)\right|^{2} \leq \frac{\varepsilon^{4}}{2}\left|w_{0}(r)\right|^{2} \max \left\{1, e^{w_{0}(r)}\right\} \\
& \leq C \varepsilon^{4}\left(\ln ^{+} r+1\right)^{2}
\end{aligned}
$$

where we used (2.10).

Now we are ready to prove the existence of the operator $\left[P_{\varepsilon}^{\prime}(0,0)\right]^{-1}$. As was mentioned earlier, we show that $P_{\varepsilon}(0,0)$ is a perturbation of the operator $A$. One problem is that although the operator $A$ is onto, its kernel is not 
empty(Actually Lemma 2.4 implies that $\operatorname{dim}\{k e r A\}=3$.). To overcome this difficulty let us decompose $Y_{\alpha}=\operatorname{ker} L \oplus(\operatorname{ker} L)^{\perp}$, and set

$$
U_{\alpha}=(\operatorname{ker} L)^{\perp} \times \mathbb{R}^{2}
$$

We equip the space $U_{\alpha}$ with the norm $\|(u, a)\|_{U_{\alpha}}=\sqrt{\|u\|_{Y_{\alpha}}^{2}+a_{1}^{2}+a_{2}^{2}}$. From now on we are going to work in the space $U_{\alpha}$ instead of $Y_{\alpha} \times \mathbb{R}^{2}$. By Theorem 2.2 there exists

$$
A^{-1}: X_{\alpha} \rightarrow U_{\alpha} \quad \forall \alpha \in\left(0, \frac{1}{2}\right)
$$

Indeed to prove (3.5) suppose $A(v, a)=0$. Then, this is equivalent to

$$
L v=-M a
$$

and Proposition 2.2 implies that $\int_{\mathbb{R}^{2}} M a \varphi_{ \pm} d x=0$. By Lemma 2.5 this, in turn, is possible only if $a=0$. So we have $L v=0$. Hence, $v=0$ by definition of the space $U_{\alpha}$.

Now we prove existence of the operator $\left[P_{\varepsilon}^{\prime}(0,0)\right]^{-1}$.

Proposition 3.1. Let $\alpha \in\left(0, \frac{1}{2}\right)$. There exists $\varepsilon_{0} \in(0,1)$ such that the inverse operator $\Gamma_{\varepsilon}=\left[P_{\varepsilon}^{\prime}(0,0)\right]^{-1} \in \mathcal{L}\left(X_{\alpha}, U_{\alpha}\right)$ exists for all $\varepsilon \in\left(0, \varepsilon_{0}\right)$. Moreover, there exists a constant $C$ such that the following inequality holds true.

$$
\left\|\Gamma_{\varepsilon}\right\|_{\mathcal{L}\left(X_{\alpha}, U_{\alpha}\right)} \leq C \quad \forall \varepsilon \in\left(0, \varepsilon_{0}\right)
$$

where $C$ is independent of $\varepsilon$.

Proof. Let us decompose

$$
P_{\varepsilon}^{\prime}(0,0)=A+\left(P_{\varepsilon}^{\prime}(0,0)-A\right)
$$

Since by (3.5) $A$ is invertible operator from $U_{\alpha}$ onto $X_{\alpha}$, it suffices to prove that there exists $\varepsilon_{0}>0$ such that

$$
\left\|P_{\varepsilon}^{\prime}(0,0)-A\right\|_{\mathcal{L}\left(U_{\alpha}, X_{\alpha}\right)}<\frac{1}{\left(\left\|A^{-1}\right\|_{\mathcal{L}\left(X_{\alpha}, U_{\alpha}\right)}+1\right)} \quad \forall \varepsilon \in\left(0, \varepsilon_{0}\right)
$$


Let $(v, b) \in Y_{\alpha} \times \mathbb{R}^{2}$ with $\|v\|_{Y_{\alpha}}+|b| \leq 1$. Then, from (2.15) and (2.17)-(2.20) we have

$$
\begin{aligned}
\left\|P_{\varepsilon}^{\prime}(0,0)[v, b]-A\left(v, b_{1}, b_{2}\right)\right\|_{X_{\alpha}} \\
\leq\left\|g_{\varepsilon}(\cdot, 0) e^{\varepsilon^{2} w_{0}} v-\rho v-2 \varepsilon^{2} g_{\varepsilon}^{2}(\cdot, 0) e^{2 \varepsilon^{2} w_{0}} v\right\|_{X_{\alpha}} \\
+\| \frac{\partial g_{\varepsilon}}{\partial a_{1}}(\cdot, 0) e^{\varepsilon^{2} w_{0}} b_{1}-\frac{\partial g_{\varepsilon}}{\partial a_{1}}(\cdot, 0) b_{1} \\
\quad-2 \varepsilon^{2} e^{2 \varepsilon^{2} w_{0}} \frac{\partial g_{\varepsilon}}{\partial a_{1}}(\cdot, 0) g_{\varepsilon}(\cdot, 0) b_{1}+4\left(\rho w_{0}-2 \rho^{2}\right) \varphi_{+} b_{1} \|_{X_{\alpha}} \\
+\| \frac{\partial g_{\varepsilon}}{\partial a_{2}}(\cdot, 0) e^{\varepsilon^{2} w_{0}} b_{2}-\frac{\partial g_{\varepsilon}(\cdot, 0)}{\partial a_{2}} b_{2} \\
\quad-2 \varepsilon^{2} e^{2 \varepsilon^{2} w_{0}} \frac{\partial g_{\varepsilon}}{\partial a_{2}}(\cdot, 0) g_{\varepsilon}(\cdot, 0) b_{2}+4\left(\rho w_{0}-2 \rho^{2}\right) \varphi_{-} b_{2} \|_{X_{\alpha}} \\
=I+J+K .
\end{aligned}
$$

Let us estimate each term of $I, J, K$ separately below. Using Lemma 3.2, we estimate $I$ as follows.

$$
\begin{aligned}
I^{2} \leq & 2 \int_{\mathbb{R}^{2}}\left|g_{\varepsilon}(z, 0)-\rho(r)\right|^{2}|v(x)|^{2}\left(1+|x|^{2+\alpha}\right) d x \\
& +2\left\|\varepsilon^{2} g_{\varepsilon}(\cdot, 0) w_{0} v\right\|_{X_{\alpha}}^{2}+2\left\|\varepsilon^{2} g_{\varepsilon}(\cdot, 0) R v\right\|_{X_{\alpha}}^{2} \\
& +2\left\|2 \varepsilon^{2} g_{\varepsilon}^{2}(\cdot, 0) e^{2 \varepsilon^{2} w_{0}} v\right\|_{X_{\alpha}}^{2}=I_{1}+I_{2}+I_{3}+I_{4},
\end{aligned}
$$

where $R$ is the function from (3.4).

As in the proof of Lemma 3.1 one can write

$$
g_{\varepsilon}(z, 0)=\frac{8(N+1)^{2}\left(r^{2 N}+\varepsilon R_{N}(\varepsilon, z)\right)}{\left(1+r^{2 N+2}+\varepsilon \tilde{R}_{N}(\varepsilon, z)\right)^{2}}
$$

where $R_{N}(\varepsilon, z)$ and $\tilde{R}_{N}(\varepsilon, z)$ satisfy the estimate

$$
\left|R_{N}(\varepsilon, z)\right| \leq C\left(1+r^{2 N-1}\right), \quad\left|\tilde{R}_{N}(\varepsilon, z)\right| \leq C\left(1+r^{2 N+1}\right), \quad r=|z|
$$

for all $\varepsilon \in(0,1)$ and $z \in \mathbb{C}$ with $C$ independent of $\varepsilon$ and $z$. Thus,

$$
\left|g_{\varepsilon}(z, 0)-\rho(|z|)\right| \leq \frac{\varepsilon C\left(1+r^{6 N+4}\right)}{\left(1+r^{8 N+8}\right)} \quad \forall \varepsilon \in(0,1) \quad z \in \mathbb{R}^{2} .
$$


Hence, thanks to Lemma 1.1, $I_{1}$ is estimated as follows.

$$
\begin{aligned}
I_{1} \leq & C \varepsilon^{2} \int_{\mathbb{R}^{2}} \frac{\left(1+|x|^{6 N+4}\right)^{2}}{\left(1+|x|^{8 N+8}\right)^{2}}\|v\|_{Y_{\alpha}}^{2}\left(\ln ^{+}|x|+1\right)^{2}\left(1+|x|^{2+\alpha}\right) d x \\
& \leq C \varepsilon^{2}\|v\|_{Y_{\alpha}}^{2}
\end{aligned}
$$

In order to estimate $I_{2}$ one can use Lemma 3.1 and (2.10) so that

$$
\begin{aligned}
I_{2} \leq & C \varepsilon^{4} \int_{\mathbb{R}^{2}} \frac{|x|^{4 N}}{\left(1+|x|^{2 N+2}\right)^{4}}\left(\ln ^{+}|x|+1\right)^{2}\|v\|_{Y_{\alpha}}^{2}\left(\ln ^{+}|x|+1\right)^{2}\left(1+|x|^{2+\alpha}\right) d x \\
& \leq C \varepsilon^{4}\|v\|_{Y_{\alpha}}^{2} .
\end{aligned}
$$

Now, using Lemma 3.1 and 3.2 , we estimate $I_{3}$ as

$$
\begin{aligned}
I_{3} \leq & C \varepsilon^{4} \int_{\mathbb{R}^{2}} \frac{|x|^{4 N}}{\left(1+|x|^{2 N+2}\right)^{4}}\|v\|_{Y_{\alpha}}^{2}\left(\ln ^{+}|x|+1\right)^{2}\left(1+|x|^{2+\alpha}\right) d x \\
& \leq C \varepsilon^{4}\|v\|_{Y_{\alpha}}^{2}
\end{aligned}
$$

and, using (2.10),

$$
\begin{aligned}
I_{4} \leq & C \varepsilon^{4} \int_{\mathbb{R}^{2}} \frac{|x|^{8 N}}{\left(1+|x|^{2 N+2}\right)^{8}}\|v\|_{Y_{\alpha}}^{2}\left(\ln ^{+}|x|+1\right)^{2}\left(1+|x|^{2+\alpha}\right) d x \\
& \leq C \varepsilon^{4}\|v\|_{Y_{\alpha}}^{2}
\end{aligned}
$$

respectively. Combining (3.11)-(3.14), we are lead to

$$
\begin{gathered}
I \leq C \varepsilon\|v\|_{Y_{\alpha}} \\
21
\end{gathered}
$$


In order to estimate $J$ we observe first

$$
\begin{aligned}
J^{2} \leq & 2\left\|\frac{\partial g_{\varepsilon}}{\partial a_{1}}(\cdot, 0)\left(\varepsilon^{2} w_{0}+\varepsilon^{4} R\right) b_{1}+4 \rho w_{0} \varphi_{+} b_{1}\right\|_{X_{\alpha}}^{2} \\
& +2\left\|2 \varepsilon^{2}\left[2 \varepsilon^{2} w_{0}+4 \varepsilon^{4} R\right] \frac{\partial g_{\varepsilon}}{\partial a_{1}}(\cdot, 0) g_{\varepsilon}(\cdot, 0) b_{1}\right\|_{X_{\alpha}}^{2} \\
& +2\left\|2 \varepsilon^{2} \frac{\partial g_{\varepsilon}}{\partial a_{1}}(\cdot, 0) g_{\varepsilon}(\cdot, 0)+8 \rho^{2} \varphi_{+} b_{1}\right\|_{X_{\alpha}}^{2} \\
\leq & 4 b_{1}^{2}\left\|\left(\varepsilon^{2} \frac{\partial g_{\varepsilon}}{\partial a_{1}}(\cdot, 0)+4 \rho \varphi_{+}\right) w_{0}\right\|_{X_{\alpha}}^{2} \\
& +4 \varepsilon^{8} b_{1}^{2}\left\|R \frac{\partial g_{\varepsilon}}{\partial a_{1}}(\cdot, 0)\right\|_{X_{\alpha}}^{2} \\
& +8 \varepsilon^{8} b_{1}^{2}\left\|\left(w_{0}+2 \varepsilon^{2} R\right) \frac{\partial g_{\varepsilon}}{\partial a_{1}}(\cdot, 0) g_{\varepsilon}(\cdot, 0)\right\|_{X_{\alpha}}^{2} \\
& +8 b_{1}^{2}\left\|\varepsilon^{2} \frac{\partial g_{\varepsilon}}{\partial a_{1}}(\cdot, 0) g_{\varepsilon}(\cdot, 0)+4 \rho^{2} \varphi_{+}\right\|_{X_{\alpha}}^{2} \\
& =J_{1}+\cdots+J_{4} .
\end{aligned}
$$

By direct computation, as in the proof of Lemma 3.1, one can write

$$
\begin{aligned}
\frac{\partial g_{\varepsilon}(z, 0)}{\partial a_{1}}= & -\frac{4}{\varepsilon^{2}} g_{\varepsilon}(z, 0) \frac{\operatorname{Re}\left[\varepsilon^{N+1} F\left(\frac{z}{\varepsilon}\right)\right]}{1+\varepsilon^{2 N+2}\left|F\left(\frac{z}{\varepsilon}\right)\right|^{2}} \\
& =-\frac{4}{\varepsilon^{2}} g_{\varepsilon}(z, 0) \frac{|z|^{N+1} \cos (N+1) \theta+\varepsilon h_{N}(\varepsilon, z)}{1+|z|^{2 N+2}+\varepsilon \tilde{h}_{N}(\varepsilon, z)}
\end{aligned}
$$

where $\left|h_{N}(\varepsilon, z)\right| \leq C\left(1+|z|^{N}\right)$, and $\left|\tilde{h}_{N}(\varepsilon, z)\right| \leq C\left(1+|z|^{2 N+1}\right)$ for all $z \in \mathbb{C}$ with $C$ independent of $\varepsilon$ and $z$.

Thus, using (3.10), we estimate

$$
\begin{aligned}
J_{1} \leq & 16 b_{1}^{2}\left\|\left(g_{\varepsilon}(\cdot, 0)-\rho\right) \frac{|z|^{N+1} \cos (N+1) \theta+\varepsilon h_{N}(\varepsilon, \cdot)}{1+|z|^{2 N+2}+\varepsilon \tilde{h}_{N}(\varepsilon, \cdot)} w_{0}\right\|_{X_{\alpha}}^{2} \\
& +16 b_{1}^{2}\left\|\left(\frac{|z|^{N+1} \cos (N+1) \theta+\varepsilon h_{N}(\varepsilon, \cdot)}{1+|z|^{2 N+2}+\varepsilon \tilde{h}_{N}(\varepsilon, \cdot)}-\varphi_{+}\right) \rho w_{0}\right\|_{X_{\alpha}}^{2} \\
\leq & C b_{1}^{2}\left\|g_{\varepsilon}(\cdot, 0)-\rho\right\|_{X_{\alpha}}^{2}+C \varepsilon^{2} b_{1}^{2}\left\|\frac{\left(1+|z|^{3 N+2}\right)}{1+|z|^{4 N+4}} \rho w_{0}\right\| \|_{X_{\alpha}}^{2} \\
\leq & C \varepsilon^{4} b_{1}^{2}+C \varepsilon^{2} b_{1}^{2} \leq C \varepsilon^{2} b_{1}^{2} .
\end{aligned}
$$


Using the estimates (3.1) - (3.4), we obtain immediately

$$
J_{2} \leq C \varepsilon^{4} b_{1}^{2}
$$

and

$$
J_{3} \leq C \varepsilon^{4} b_{1}^{2} .
$$

To estimate $J_{4}$ let us begin first

$$
J_{4} \leq 8 b_{1}^{2}\left(\left\|\varepsilon^{2} \frac{\partial g_{\varepsilon}}{\partial a_{1}}(\cdot, 0)\left(g_{\varepsilon}(\cdot, 0)-\rho\right)\right\|_{X_{\alpha}}^{2}+\left\|\left(\varepsilon^{2} \frac{\partial g_{\varepsilon}}{\partial a_{1}}(\cdot, 0)+4 \rho \varphi_{+}\right) \rho\right\|_{X_{\alpha}}^{2}\right) .
$$

By (2.1), (3.2)

$$
\begin{aligned}
J_{4} \leq & C b_{1}^{2}\left(\left\|g_{\varepsilon}(\cdot, 0)-\rho\right\|_{X_{\alpha}}^{2}+\left\|\varepsilon^{2} \frac{\partial g_{\varepsilon}}{\partial a_{1}}(\cdot, 0)+4 \rho \varphi_{+}\right\|_{X_{\alpha}}^{2}\right) \\
& \leq C \varepsilon^{2} b_{1}^{2}
\end{aligned}
$$

where in the last inequality we used (3.11) with $v \equiv 1$ and (3.17) with $b_{1}=1$.

Combining (3.16)-(3.19), (3.21) we obtain

$$
J \leq C \varepsilon b_{1} .
$$

Estimate of $K$ is similar to $J$, and we have easily

$$
K \leq C \varepsilon b_{2}
$$

Combining (3.15), (3.22) and (3.23), we finally estimate

$$
\left\|P_{\varepsilon}^{\prime}(0,0)[v, b]-A\left(v, b_{1}, b_{2}\right)\right\|_{X_{\alpha}} \leq C \varepsilon\left(\|v\|_{Y_{\alpha}}+|b|\right) \leq C \varepsilon .
$$

Taking $\varepsilon_{0}=\frac{1}{2 C\left(\left\|A^{-1}\right\|_{\mathcal{L}\left(X_{\alpha}, U_{\alpha}\right)}+1\right)},(3.7)$ is proved.

As it was mentioned earlier, to apply the Newton-Kantorovich theorem we will choose $v_{0}=0 \in U_{\alpha}$. The following proposition provides the estimate for the parameter $\eta$ of $(i i)$ in Theorem 2.1. 
Proposition 3.2. Let $\alpha \in\left(0, \frac{1}{2}\right)$, then there exists $\varepsilon_{1}>0$ such that

$$
\left\|P_{\varepsilon}(0,0)\right\|_{X_{\alpha}} \leq C \varepsilon^{3} \quad \forall \varepsilon \in\left(0, \varepsilon_{1}\right) .
$$

Proof. By virtue of Lemma 3.2, and using the definition of the function $w_{0}$, one can rewrite (2.14) as follows.

$$
\begin{aligned}
P_{\varepsilon}(0,0)= & g_{\varepsilon}(z, 0)\left(1+\varepsilon^{2} w_{0}+\varepsilon^{4} R_{1}(|x|)\right)-\varepsilon^{2} g_{\varepsilon}^{2}(z, 0)\left(1+2 \varepsilon^{2} w_{0}+\varepsilon^{4} \tilde{R}_{1}(|x|)\right) \\
& \quad-g_{\varepsilon}(z, 0)+\varepsilon^{2}\left(-\rho w_{0}+\rho^{2}\right) \\
= & \varepsilon^{2}\left(g_{\varepsilon}(z, 0)-\rho\right) w_{0}+\varepsilon^{2}\left(\rho^{2}-g_{\varepsilon}^{2}(z, 0)\right)-2 \varepsilon^{4} g_{\varepsilon}^{2}(z, 0) w_{0} \\
& +\varepsilon^{4} g_{\varepsilon}(z, 0) R_{1}(|x|)-\varepsilon^{6} g_{\varepsilon}^{2}(z, 0) \tilde{R}_{1}(|x|),
\end{aligned}
$$

where $\left|R_{1}(|x|)\right|+\left|\tilde{R}_{1}(|x|)\right| \leq C\left(\ln ^{+}|x|+1\right)^{2}$. We thus have

$$
\begin{aligned}
\left\|P_{\varepsilon}(0,0)\right\|_{X_{a}}^{2} \leq & 2 \varepsilon^{4} \int_{\mathbb{R}^{2}}\left|g_{\varepsilon}(z, 0)-\rho\right|^{2}\left|w_{0}\right|^{2}\left(1+|x|^{2+\alpha}\right) d x \\
& +4 \varepsilon^{4} \int_{\mathbb{R}^{2}}\left|g_{\varepsilon}(z, 0)-\rho\right|^{2}\left(|\rho|^{2}+\left|g_{\varepsilon}(z, 0)\right|^{2}\right)\left(1+|x|^{2+\alpha}\right) d x \\
& +8 \varepsilon^{8} \int_{\mathbb{R}^{2}}\left|g_{\varepsilon}(z, 0)\right|^{4}\left|w_{0}\right|^{2}\left(1+|x|^{2+\alpha}\right) d x \\
& +C \varepsilon^{8} \int_{\mathbb{R}^{2}}\left|g_{\varepsilon}(z, 0)\right|^{2}\left(1+|x|^{2+\alpha}\right)\left(\ln ^{+}|x|+1\right)^{4} d x \\
& +C \varepsilon^{12} \int_{\mathbb{R}^{2}}\left|g_{\varepsilon}(z, 0)\right|^{4}\left(1+|x|^{2+\alpha}\right)\left(\ln ^{+}|x|+1\right)^{4} d x
\end{aligned}
$$

Using the previous estimates (3.10) and (3.1), we obtain easily

$$
\left\|P_{\varepsilon}(0,0)\right\|_{X_{\alpha}}^{2} \leq C \varepsilon^{6}+C \varepsilon^{6}+C \varepsilon^{8}+C \varepsilon^{8}+C \varepsilon^{12} \leq C \varepsilon^{6}
$$

for all sufficiently small $\varepsilon$. This completes the proof of the proposition.

To apply Theorem 2.1 in the next section we are going to choose $v_{0}=0$ and $\tilde{r}=\varepsilon^{2}$. Thus the following proposition provides the necessary estimate for the parameter $K$ of $(i i i)$ in Theorem 2.1 . 
Proposition 3.3. Let $\alpha \in\left(0, \frac{1}{2}\right)$. Then, there exist $\varepsilon_{0} \in(0,1)$ such that for all $\varepsilon \in\left(0, \varepsilon_{0}\right)$ and $(u, a) \in U_{\alpha}$ with $\|(u, a)\|_{U_{\alpha}} \leq \varepsilon^{2}$ we have the estimate

$$
\left\|P_{\varepsilon}^{\prime \prime}(u, a)\right\|_{\mathcal{L}\left(U_{\alpha} \times U_{\alpha} ; X_{\alpha}\right)} \leq \frac{C}{\varepsilon^{2}}
$$

where constant $C$ is independent of $\varepsilon$.

Proof. Let us assume $\|(u, a)\|_{U_{\alpha}} \leq \varepsilon^{2}$ below. From (2.16) we have

$$
\begin{aligned}
& \left\|P^{\prime \prime}(u, a)[v, b ; \tilde{v}, \tilde{b}]\right\|_{X_{\alpha}} \\
& \leq\left\|g_{\varepsilon} e^{u+\varepsilon^{2} w_{0}} v \tilde{v}\right\|_{X_{\alpha}}+\sum_{j=1}^{2}\left\|\frac{\partial g_{\varepsilon}}{\partial a_{j}} e^{u+\varepsilon^{2} w_{0}} b_{j} \tilde{v}\right\|_{X_{\alpha}} \\
& +\sum_{j=1}^{2}\left\|\frac{\partial g_{\varepsilon}}{\partial a_{j}} e^{u+\varepsilon^{2} w_{0}} v \tilde{b}_{j}\right\|_{X_{\alpha}} \\
& +\sum_{j, k=1}^{2}\left\|\frac{\partial^{2} g_{\varepsilon}}{\partial a_{j} \partial a_{k}}\left(e^{u+\varepsilon^{2} w_{0}}-1\right) b_{j} \tilde{b}_{k}\right\|_{X_{\alpha}} \\
& +4 \varepsilon^{2} \sum_{j=1}^{2}\left\|\frac{\partial g_{\varepsilon}}{\partial a_{j}} g_{\varepsilon} e^{2 u+2 \varepsilon^{2} w_{0}} b_{j} \tilde{v}\right\|_{X_{\alpha}} \\
& +2 \varepsilon^{2} \sum_{j, k=1}^{2}\left\|\left(\frac{\partial^{2} g_{\varepsilon}}{\partial a_{j} \partial a_{k}} g_{\varepsilon}+\frac{\partial g_{\varepsilon}}{\partial a_{j}} \frac{\partial g_{\varepsilon}}{\partial a_{k}}\right) b_{j} \tilde{b}_{k} e^{2 u+2 \varepsilon^{2} w_{0}}\right\|_{X_{\alpha}} \\
& +4 \varepsilon^{2}\left\|g_{\varepsilon}^{2} e^{2 u+2 \varepsilon^{2} w_{0}} v \tilde{v}\right\|_{X_{\alpha}} \\
& +4 \varepsilon^{2} \sum_{j=1}^{2}\left\|\frac{\partial g_{\varepsilon}}{\partial a_{j}} g_{\varepsilon} e^{2 u+2 \varepsilon^{2} w_{0}} v \tilde{b}_{j}\right\|_{X_{\alpha}}=I_{1}+I_{2}+\cdots+I_{8} .
\end{aligned}
$$

Below each term of $I_{1} \cdots I_{8}$ will be estimated separately. To estimate of $I_{1}$ we use (2.10) and (1.3) as follows.

$$
\begin{aligned}
I_{1}^{2} & =\int_{\mathbb{R}^{2}}\left|g_{\varepsilon}(z, a)\right|^{2} e^{2 u+2 \varepsilon^{2} w_{0}}|v|^{2}|\tilde{v}|^{2}\left(1+|x|^{2+\alpha}\right) d x \\
& \leq C \int_{0}^{\infty} \frac{r^{4 N}}{\left(1+r^{2 N+2}\right)^{4}} e^{2 C_{1}\|u\|_{Y_{\alpha}}\left(\ln ^{+} r+1\right)}\|v\|_{Y_{\alpha}}^{2}\|\tilde{v}\|_{Y_{\alpha}}^{2}\left(\ln ^{+} r+1\right)^{4}\left(1+r^{2+\alpha}\right) r d r \\
& \leq C\|v\|_{Y_{\alpha}}^{2}\|\tilde{v}\|_{Y_{\alpha}}^{2} \int_{0}^{\infty} \frac{\left(1+r^{2+\alpha+2 \varepsilon^{2} C_{1}+4 N+1}\right)\left(\ln ^{+} r+1\right)^{4}}{\left(1+r^{2 N+2}\right)^{4}} d r \\
& \leq C\|v\|_{Y_{a}}^{2}\|\tilde{v}\|_{Y_{\alpha}}^{2},
\end{aligned}
$$


where $\varepsilon_{0}$ was chosen so that

$$
4 \varepsilon_{0}^{2} C_{1}<3,
$$

where $C_{1}$ is the constant from the inequality (1.3). Using (3.2), (2.9) one estimate $I_{2}$,

$$
\begin{aligned}
I_{2}^{2} & \leq 2 \sum_{j=1}^{2} \int_{\mathbb{R}^{2}}\left|\frac{\partial g_{\varepsilon}(z, a)}{\partial a_{j}}\right|^{2} e^{2 u+2 \varepsilon^{2} w_{0}}\left|b_{j}\right|^{2}|\tilde{v}|^{2}\left(1+|x|^{2+\alpha}\right) d x \\
& \leq \frac{C}{\varepsilon^{4}} \int_{0}^{\infty} \frac{r^{4 N}}{\left(1+r^{2 N+2}\right)^{4}} e^{2 C_{1}\|u\|_{Y_{\alpha}}\left(\ln ^{+} r+1\right)}|b|^{2}\|\tilde{v}\|_{Y_{\alpha}}^{2}\left(\ln ^{+} r+1\right)^{2}\left(1+r^{2+\alpha}\right) r d r \\
& \leq \frac{C}{\varepsilon^{4}}|b|^{2}\|\tilde{v}\|_{Y_{\alpha}}^{2} \int_{0}^{\infty} \frac{\left(1+r^{2+\alpha+2 \varepsilon^{2} C_{1}+4 N+1}\right)\left(\ln ^{+} r+1\right)^{2}}{\left(1+r^{2 N+2}\right)^{4}} d r \\
& \leq \frac{C}{\varepsilon^{4}}|b|^{2}\|\tilde{v}\|_{Y_{\alpha}}^{2},
\end{aligned}
$$

where we used the assumption on $\varepsilon_{0}$ in (3.31). Estimate of $I_{3}$ is similar to $I_{2}$, and we obtain

$$
I_{3} \leq \frac{C}{\varepsilon^{2}}|\tilde{b}|\|v\|_{Y_{\alpha}} .
$$

Thanks to (3.3) and (2.4), (2.10), (1.3) $I_{4}$ can be estimated as follows.

$$
\begin{aligned}
I_{4}^{2} & \leq 2 \sum_{j, k=2}^{2} \int_{\mathbb{R}^{2}}\left|\frac{\partial^{2} g_{\varepsilon}(z, a)}{\partial a_{j} \partial a_{k}}\right|^{2}\left(e^{u+\varepsilon^{2} w_{0}}-1\right)^{2}\left|b_{j}\right|^{2}\left|\tilde{b}_{k}\right|^{2}\left(1+|x|^{2+\alpha}\right) d x \\
& \leq \frac{C}{\varepsilon^{8}} \int_{\mathbb{R}^{2}} \frac{|x|^{4 N}\left|u+\varepsilon^{2} w_{0}\right|^{2} e^{2|u|}\left\|e^{\varepsilon^{2} w_{0}}\right\|_{C^{0}\left(R_{+}^{1}\right)}^{2}|b|^{2}|\tilde{b}|^{2}\left(1+|x|^{2+\alpha}\right)}{\left(1+|x|^{2 N+2}\right)^{4}} d x \\
& \leq \frac{C}{\varepsilon^{8}} \int_{0}^{\infty} \frac{r^{4 N} \varepsilon^{4}\left(\ln ^{+} r+1\right)^{2} e^{2 \varepsilon^{2} C_{1}\left(\ln ^{+} r+1\right)}|b|^{2}|\tilde{b}|^{2}\left(1+r^{2+\alpha}\right)}{\left(1+r^{2 N+2}\right)^{4}} r d r \\
& \leq \frac{C}{\varepsilon^{4}}|b|^{2}|\tilde{b}|^{2} \int_{0}^{\infty} \frac{\left(1+r^{2+\alpha+2 \varepsilon^{2} C_{1}+4 N+1}\right)\left(\ln ^{+} r+1\right)^{2}}{\left(1+r^{2 N+2}\right)^{4}} d r \\
& \leq \frac{C}{\varepsilon^{4}}|b|^{2}|\tilde{b}|^{2},
\end{aligned}
$$

where the mean value theorem was used in the second inequality, and (3.31) was used in the last inequality. Next, we estimate $I_{5}$ and $I_{6}$, using (3.1)- 
$(3.3),(2.10)$ as follows.

$$
\begin{aligned}
I_{5}^{2} & \leq 32 \varepsilon^{4} \sum_{i=1}^{2} \int_{\mathbb{R}^{2}}\left|\frac{\partial g_{\varepsilon}(z, a)}{\partial a_{j}}\right|^{2}\left|g_{\varepsilon}(z, a)\right|^{2} e^{4 u+4 \varepsilon^{2} w_{0}}\left|b_{j}\right|^{2}|\tilde{v}|^{2}\left(1+|x|^{2+\alpha}\right) d x \\
& \leq C \int_{0}^{\infty} \frac{r^{8 N}}{\left(1+r^{2 N+2}\right)^{8}} e^{4 C_{1}\|u\|_{Y_{\alpha}}\left(\ln ^{+} r+1\right)}|b|^{2}\|\tilde{v}\|_{Y_{\alpha}}^{2}\left(\ln ^{+} r+1\right)^{2}\left(1+r^{2+\alpha}\right) r d r \\
& \leq C|b|^{2}\|\tilde{v}\|_{Y_{\alpha}}^{2} \int_{0}^{\infty} \frac{\left\{1+r^{2+\alpha+8 N+4 C_{1} \varepsilon_{0}^{2}+1}\right\}\left(\ln ^{+} r+1\right)^{2}}{\left(1+r^{2 N+2}\right)^{8}} d r \\
& \leq C|b|^{2}\|\tilde{v}\|_{Y_{\alpha}}^{2},
\end{aligned}
$$

and

$$
\begin{aligned}
I_{6}^{2} \leq 8 \varepsilon^{4} \sum_{i, k=1}^{2} \int_{\mathbb{R}^{2}}\left(\left|\frac{\partial^{2} g_{\varepsilon}(z, a)}{\partial a_{j} \partial a_{k}}\right|^{2}\left|g_{\varepsilon}(z, a)\right|^{2}+\left|\frac{\partial g_{\varepsilon}(z, a)}{\partial a_{j}}\right|^{2}\left|\frac{\partial g_{\varepsilon}(z, a)}{\partial a_{k}}\right|^{2}\right) \\
\quad \cdot\left|b_{j}\right|^{2}\left|\tilde{b}_{k}\right|^{2} e^{4 u+4 \varepsilon^{2} w_{0}}\left(1+|x|^{2+\alpha}\right) d x \\
\leq \frac{C|b|^{2}|\tilde{b}|^{2}}{\varepsilon^{4}} \int_{0}^{\infty} \frac{r^{8 N}}{\left(1+r^{2 N+2}\right)^{8}} e^{4 C_{1}\|u\|_{Y_{\alpha}}\left(\ln ^{+} r+1\right)}\left(1+r^{2+\alpha}\right) r d r \\
\leq \frac{C}{\varepsilon^{4}}|b|^{2}|\tilde{b}|^{2} \int_{0}^{\infty} \frac{1+r^{2+\alpha+4 C_{1} \varepsilon_{0}^{2}+8 N+1}}{\left(1+r^{2 N+2}\right)^{8}} d r \leq \frac{C}{\varepsilon^{4}}|b|^{2}|\tilde{b}|^{2} .
\end{aligned}
$$

Estimate of $I_{7}$ is similar to $I_{1}$, and we have

$$
I_{7} \leq C \varepsilon^{2}\|v\|_{Y_{\alpha}}\|\tilde{v}\|_{Y_{\alpha}}
$$

Estimate of $I_{8}$ is similar to $I_{5}$, and we have

$$
I_{8} \leq C|\tilde{b}|\|v\|_{Y_{\alpha}}
$$

Estimates (3.30), (3.32)-(3.38) provides us with (3.28).

\section{Proof of the Main Theorem.}

We are now equipped with all the necessary facts to apply Theorem 2.1 to prove our main theorem.

Proof of the Main Theorem. We apply Theorem 2.1 to our functional equation (2.12). Set $B_{1}=U_{\frac{1}{8}}, B_{2}=X_{\frac{1}{8}}$, and $v_{0}=0$. Also, we set $\alpha=\frac{1}{8}$ in 
Propositions 3.1-3.3. We denote $v=(u, a)$, and define $\mathbf{P}_{\varepsilon}(v)=P_{\varepsilon}(u, a)$. Then by Proposition 3.1 there exists the operator $\Gamma_{\varepsilon}=\left[\mathbf{P}_{\varepsilon}^{\prime}(0)\right]^{-1}$ with its norm estimate given by (3.6). Combining Proposition 3.1 with Proposition 3.2 , we obtain

$$
\left\|\Gamma_{\varepsilon} \mathbf{P}_{\varepsilon}(0)\right\|_{U_{\frac{1}{8}}} \leq\left\|\Gamma_{\varepsilon}\right\|_{\mathcal{L}\left(X_{\frac{1}{8}}, U_{\frac{1}{8}}\right)}\left\|\mathbf{P}_{\varepsilon}(0)\right\|_{X_{\frac{1}{8}}} \leq C \varepsilon^{3} \equiv \eta(\varepsilon)
$$

for all $\varepsilon \in\left(0, \varepsilon_{0}\right)$. On the other hand, combining Proposition 3.1 with Proposition 3.3 , we have

$$
\left\|\Gamma_{\varepsilon} \mathbf{P}_{\varepsilon}^{\prime \prime}(v)\right\|_{U_{\frac{1}{8}}} \leq\left\|\Gamma_{\varepsilon}\right\|_{\mathcal{L}\left(X_{\frac{1}{8}}, U_{\frac{1}{8}}\right)}\left\|\mathbf{P}_{\varepsilon}^{\prime \prime}(v)\right\| \leq C \varepsilon^{-2} \equiv K(\varepsilon)
$$

for all $v \in \Omega_{0}=\left\{v \in U_{\frac{1}{8}} \mid\|v\|_{U_{\frac{1}{8}}} \leq \varepsilon^{2}\right\}$ and $\varepsilon \in\left(0, \varepsilon_{0}\right)$. Hence

$$
h(\varepsilon)=K(\varepsilon) \eta(\varepsilon) \leq C \varepsilon \quad \forall \varepsilon \in\left(0, \varepsilon_{0}\right) .
$$

Taking the parameter $\varepsilon$ sufficiently small, we have $h(\varepsilon)<\frac{1}{2}$. Moreover, since $\tilde{r}=\varepsilon^{2}$, and $r_{0}=O\left(\varepsilon^{3}\right)$ as $\varepsilon \rightarrow 0$, we have $\tilde{r}>r_{0}$ for all sufficiently small $\varepsilon$. Thus all conditions of Theorem 2.1 are satisfied, and there exists a solution, $v_{\varepsilon}^{*}=\left(u_{\varepsilon}^{*}, a_{\varepsilon}^{*}\right) \in \Omega_{0}$ to the equation $\mathbf{P}_{\varepsilon}(v)=0$ such that

$$
\left\|v_{\varepsilon}^{*}\right\|_{U_{\frac{1}{8}}} \leq \frac{1-\sqrt{1-2 h}}{h} \eta \leq C \varepsilon^{3}
$$

where $C$ is independent of $\varepsilon$.

We now verify the decay estimate (11) for $u$ recovered from formula (2.13), thus showing that our solution $u$ is really nontopological. From the explicit formula given in (2.1) we know that

$$
\ln \rho_{\varepsilon, a_{\varepsilon}^{*}(x)}=-(2 N+4) \ln |x|+o(\ln |x|) \quad \text { as }|x| \rightarrow \infty .
$$

From (1.3) and (4.2) we obtain

$$
\begin{aligned}
\left|u_{\varepsilon}^{*}(x)\right| & \leq C\left\|u_{\varepsilon}^{*}\right\|_{Y_{\frac{1}{8}}}\left(\ln ^{+}|x|+1\right) \\
& \leq C\left\|v_{\varepsilon}^{*}\right\|_{U_{\frac{1}{8}}}\left(\ln ^{+}|x|+1\right) \\
& \leq C \varepsilon^{3}\left(\ln ^{+}|x|+1\right) .
\end{aligned}
$$

This, implies then

$$
\left|u_{\varepsilon}^{*}(\varepsilon x)\right| \leq C \varepsilon^{3}\left(\ln ^{+}|\varepsilon x|+1\right) \leq C \varepsilon^{3}\left(\ln ^{+}|x|+1\right) .
$$


Also, from the asymptotic formula (2.10)

$$
\varepsilon^{2} w_{0}(\varepsilon x)=-\tilde{C} \varepsilon^{2} \ln |x|+o(\ln |x|) \quad \text { as }|x| \rightarrow \infty,
$$

where $\tilde{C}$ is a positive number. Taking into account (4.3)-(4.5), we deduce from (2.13) that there is $\varepsilon_{1} \in\left(0, \varepsilon_{0}\right)$, and a constant $\beta=\beta(\varepsilon)>0$ such that our solution $u(x)$ of (9) satisfies

$$
u(x)=-(2 N+4+\beta) \ln |x|+o(\ln |x|) \quad \text { as }|x| \rightarrow \infty
$$

for all $\varepsilon \in\left(0, \varepsilon_{1}\right)$. Then,

$$
e^{u(x)}=O\left(\frac{1}{|x|^{2 N+4+\beta}}\right) \quad \text { as }|x| \rightarrow \infty .
$$

We recall that $z=x_{1}+i x_{2}, \partial_{z}=\frac{1}{2}\left(\frac{\partial}{\partial x_{1}}-i \frac{\partial}{\partial x_{2}}\right), \bar{\partial}_{z}=\frac{1}{2}\left(\frac{\partial}{\partial x_{1}}+i \frac{\partial}{\partial x_{2}}\right)$ as previously, and define

$$
\phi(z)=\exp \frac{1}{2}(u+i \theta) \text { with } \theta=\sum_{j=1}^{N} 2 \arg \left(z-z_{j}\right)
$$

and

$$
A_{1}=-\operatorname{Re}\left\{2 i \bar{\partial}_{z} \ln \phi(z)\right\}, A_{2}=-\operatorname{Im}\left\{2 i \bar{\partial}_{z} \ln \phi(z)\right\} .
$$

Then, $(\phi, A)$ becomes a solution of the Bogomol'nyi equations (4)-(5) satisfying (7). We now show that our solution $(\phi, A)$ is of finite energy, and satisfies the decay estimates (11). Since by (4.7) and (4.8)

$$
|\phi(x)|^{2}=O\left(\frac{1}{|x|^{2 N+4+\beta}}\right) \text { as }|x| \rightarrow \infty,
$$

we obtain

$$
|\phi|^{2}\left(1-|\phi|^{2}\right) \in L^{1}\left(\mathbb{R}^{2}\right)
$$

Then by (5) and (4.9)

$F_{12}(x)=O\left(\frac{1}{|x|^{2 N+4+\beta}}\right) \quad$ as $|x| \rightarrow \infty$, and $\frac{F_{12}^{2}}{|\phi|^{2}},|\phi|^{2}\left(1-|\phi|^{2}\right) \in L^{1}\left(\mathbb{R}^{2}\right)$.

Now it suffices to show that

$$
\left|D_{1} \phi\right|^{2}+\left|D_{2} \phi\right|^{2}=O\left(\frac{1}{|x|^{2 N+4+\beta}}\right) \text { as }|x| \rightarrow \infty,
$$


and also that it is integrable. From (4) we have immediately that $\bar{\partial}_{z} \ln \phi(z)=$ $-i \bar{\alpha}$, where $\bar{\alpha}=\frac{1}{2}\left(A_{1}+i A_{2}\right)$, which, in turn, gives

$$
\frac{1}{2} \frac{\partial \theta}{\partial x_{1}}-A_{1}=-\frac{1}{2} \frac{\partial u}{\partial x_{2}}, \frac{1}{2} \frac{\partial \theta}{\partial x_{2}}-A_{2}=\frac{1}{2} \frac{\partial u}{\partial x_{1}} .
$$

Thus, $D_{1} \phi=\left(\frac{1}{2} \frac{\partial u}{\partial x_{1}}+\frac{i}{2} \frac{\partial \theta}{\partial x_{1}}-i A_{1}\right) \phi=\frac{1}{2}\left(\frac{\partial u}{\partial x_{1}}-i \frac{\partial u}{\partial x_{2}}\right) \phi$ and $D_{2} \phi=\left(\frac{1}{2} \frac{\partial u}{\partial x_{2}}+\right.$ $\left.\frac{i}{2} \frac{\partial \theta}{\partial x_{2}}-i A_{2}\right) \phi=\frac{1}{2}\left(\frac{\partial u}{\partial x_{2}}+i \frac{\partial u}{\partial x_{1}}\right) \phi$. Therefore,

$$
\left|D_{1} \phi\right|^{2}+\left|D_{2} \phi\right|^{2} \leq \frac{1}{2}|\nabla u|^{2}|\phi|^{2}=\frac{1}{2}|\nabla u|^{2} e^{u} .
$$

From (9) and Proposition 1.1 we obtain

$$
u(x)=\frac{1}{2 \pi} \int_{\mathbb{R}^{2}} \ln |x-y| e^{u(y)}\left(e^{u(y)}-1\right) d y+2 \sum_{j=1}^{N} \ln \left|x-z_{j}\right|+C
$$

for some constant $C$. Since $u(x) \leq 0$ for $x \in \mathbb{R}^{2}$ (by the maximum principle applied to (9)), taking derivative of $u$ from (4.12), and using the inequality $\left(\sum_{j=1}^{n} a_{j}\right)^{2} \leq n \sum_{j=1}^{n} a_{j}^{2}$, we obtain

$$
|\nabla u(x)|^{2} e^{u(x)} \leq C e^{u(x)}\left(\int_{\mathbb{R}^{2}} \frac{e^{u(y)}}{|x-y|} d y\right)^{2}+C \sum_{j=1}^{N} \frac{e^{u(x)}}{\left|x-z_{j}\right|^{2}}=I_{1}(x)+I_{2}(x) .
$$

Since $u(x)=2 \ln \left|x-z_{j}\right|+O(1)$ as $x \rightarrow z_{j}$, the function $I_{2}(x)$ is locally integrable, and by (4.7)

$$
I_{2}(x)=O\left(\frac{1}{|x|^{2 N+4+\beta}}\right) \text { as }|x| \rightarrow \infty .
$$

Hence $I_{2}(x) \in L^{1}\left(\mathbb{R}^{2}\right)$.

From the estimate

$$
\int_{\mathbb{R}^{2}} \frac{e^{u(y)}}{|x-y|} d y \leq \int_{|x-y| \leq 1} \frac{e^{u(y)}}{|x-y|} d y+\int_{|x-y|>1} e^{u(y)} d y \leq C,
$$

and (4.7) we also find that

$$
I_{1}(x)=O\left(\frac{1}{|x|^{2 N+4+\beta}}\right) \quad \text { as }|x| \rightarrow \infty
$$


and is also integrable. We now prove (12). From (5) with $\kappa=2$ we first obtain

$$
\int_{\mathbb{R}^{2}} F_{12} d x=-\frac{1}{2} \int_{\mathbb{R}^{2}} e^{u}\left(e^{u}-1\right) d x
$$

Let us denote $B\left(z_{j}, \delta\right)=\left\{z \in \mathbb{C}^{1}|| z-z_{j} \mid<\delta\right\}$, and

$$
B_{R}=\left\{z \in \mathbb{C}^{1}|| z \mid<R\right\}, \quad S_{R}=\left\{z \in \mathbb{C}^{1}|| z \mid=R\right\} .
$$

Let us choose $\delta$ small enough so that $B\left(z_{j}, \delta\right)(j=1, \cdots, N)$ 's are mutually disjoint and $R$ large enough so that $B_{R}$ contains all of $B\left(z_{j}, \delta\right)$ 's. Integrating (9) over $B_{R} \backslash \cup_{j=1}^{N} B\left(z_{j}, \delta\right)$, we obtain by the divergence theorem

$$
\oint_{S_{R}} \frac{\partial u}{\partial r} d s=\int_{B_{R} \backslash \cup_{j=1}^{N} B\left(z_{j}, \delta\right)} e^{u}\left(e^{u}-1\right) d x+\sum_{j=1}^{N} \oint_{\left|x-z_{j}\right|=\delta} \frac{x-z_{j}}{\left|x-z_{j}\right|} \cdot \nabla u d s
$$

Now, using the representation formula of $u(x)$ in (4.12), we deduce

$$
\begin{aligned}
\oint_{\left|x-z_{j}\right|=\delta} \frac{x-z_{j}}{\left|x-z_{j}\right|} \cdot \nabla u d s & =2 \oint_{\left|x-z_{j}\right|=\delta} \frac{x-z_{j}}{\left|x-z_{j}\right|} \cdot \nabla \ln \left|x-z_{j}\right| d s+o(1) \\
& =2 \oint_{|x|=\delta} \frac{\partial}{\partial r} \ln r d s+o(1)=4 \pi+o(1)
\end{aligned}
$$

as $\delta \rightarrow 0$. Substituting (4.14) into (4.13), and passing $\delta \rightarrow 0$, we find that

$$
\oint_{S_{R}} \frac{\partial u}{\partial r} d s=\int_{B_{R}} e^{u}\left(e^{u}-1\right) d x+4 \pi N
$$

From (4.12) and (4.15)

$$
\int_{\mathbb{R}^{2}} F_{12} d x=-\frac{1}{2} \lim _{R \rightarrow \infty} \oint_{S_{R}} \frac{\partial u}{\partial r} d s+2 \pi N
$$

and for our solution, $u(x)=u_{\varepsilon}(x)$ given by (2.13), it suffices to show that there exists a positive constant $\hat{C}$ such that

$$
-\frac{1}{2} \lim _{R \rightarrow \infty} \oint_{S_{R}} \frac{\partial u_{\varepsilon}}{\partial r} d s=2 \pi N+4+\hat{C} \varepsilon^{2}+O\left(\varepsilon^{3}\right)
$$


as $\varepsilon \rightarrow 0$. Taking into account the formula for $u_{\varepsilon}(x)$ in $(2.13)$, it suffices, in turn, to prove the followings, (4.17)-(4.19).

$$
\begin{gathered}
-\frac{1}{2} \lim _{R \rightarrow \infty} \oint_{S_{R}} \frac{\partial}{\partial r} \ln \rho_{\varepsilon, a_{\varepsilon}^{*}} d s=2 \pi N+4, \\
-\lim _{R \rightarrow \infty} \oint_{S_{R}} \frac{\partial w_{0}(\varepsilon|z|)}{\partial r} d s=\hat{C}
\end{gathered}
$$

where $\hat{C}$ is a positive constant independent of $\varepsilon$, and

$$
\lim _{R \rightarrow \infty} \oint_{S_{R}} \frac{\partial u_{\varepsilon}^{*}(\varepsilon z)}{\partial r} d s=O\left(\varepsilon^{3}\right)
$$

as $\varepsilon \rightarrow 0$. We first prove (4.17). From the formula (2.1) one can write

$$
\begin{aligned}
\frac{\partial}{\partial r} \ln \rho_{\varepsilon, a_{\varepsilon}^{*}}= & \frac{1}{\rho_{\varepsilon, a_{\varepsilon}^{*}}} \frac{\partial}{\partial r} \rho_{\varepsilon, a_{\varepsilon}^{*}} \\
= & \frac{8 \varepsilon^{2 N+2}}{\rho_{\varepsilon, a_{\varepsilon}^{*}}}\left\{\frac{\frac{\partial}{\partial r}|f(z)|^{2}}{\left(1+\varepsilon^{2 N+2}\left|F(z)+\frac{a_{\varepsilon}^{*}}{\varepsilon^{N+3}}\right|^{2}\right)^{2}}\right. \\
& \left.\quad-\frac{2|f(z)|^{2} \varepsilon^{2 N+2} \frac{\partial}{\partial r}\left|F(z)+\frac{a_{\varepsilon}^{*}}{\varepsilon^{N+3}}\right|^{2}}{\left(1+\varepsilon^{2 N+2}\left|F(z)+\frac{a_{\varepsilon}^{*}}{\varepsilon^{N+3}}\right|^{2}\right)^{3}}\right\}
\end{aligned}
$$

with

$$
|f(z)|^{2}=(N+1)^{2} r^{2 N}+p_{2 N-1}(\theta) r^{2 N-1}+\cdots+p_{0}(\theta)
$$

and

$$
|F(z)|^{2}=r^{2 N+2}+q_{2 N+1}(\theta) r^{2 N+1}+\cdots+q_{0}(\theta),
$$

where $p_{j}(\theta)$ 's and $q_{k}(\theta)$ 's are functions only of $\theta$. Thus, substituting

$$
\frac{\partial}{\partial r}|f(z)|^{2}=2 N(N+1)^{2} r^{2 N-1}+(2 N-1) p_{2 N-1}(\theta) r^{2 N-2}+\cdots+p_{1}(\theta)
$$

and

$$
\frac{\partial}{\partial r}|F(z)|^{2}=(2 N+2) r^{2 N+1}+(2 N+1) q_{2 N+1}(\theta) r^{2 N}+\cdots+q_{1}(\theta)
$$


into (4.20), we have for each $\varepsilon>0$

$$
\begin{aligned}
\oint_{S_{R}} & \frac{\partial}{\partial r} \ln \rho_{\varepsilon, a_{\varepsilon}^{*}} d s \\
\quad= & \oint_{S_{R}} \frac{1}{|f(z)|^{2}}\left\{\frac{\partial}{\partial r}|f(z)|^{2}-\frac{2|f(z)|^{2} \varepsilon^{2 N+2} \frac{\partial}{\partial r}\left|F(z)+\frac{a_{\varepsilon}^{*}}{\varepsilon^{N+3}}\right|^{2}}{1+\varepsilon^{2 N+2}\left|F(z)+\frac{a_{\varepsilon}^{*}}{\varepsilon^{N+3}}\right|^{2}}\right\} d s \\
\quad= & \int_{0}^{2 \pi}\left\{\frac{2 N(N+1)^{2} R^{2 N-1}}{(N+1)^{2} R^{2 N}}-\frac{2(2 N+2) \varepsilon^{2 N+2} R^{2 N+1}}{1+\varepsilon^{2 N+2} R^{2 N+2}}\right\} R d \theta+O\left(\frac{1}{R}\right) \\
= & -4 \pi N-8 \pi+O\left(\frac{1}{R}\right)
\end{aligned}
$$

as $R \rightarrow \infty$. Thus (4.17) is proved. Next, in order to prove (4.18) we first note

$$
r \frac{\partial w_{0}(r)}{\partial r}=\int_{0}^{\infty} \varphi_{0}(t) t \rho^{2}(t) d t+O\left(\frac{1}{r}\right) \quad \text { as } r \rightarrow \infty
$$

which is obtained by explicit computation, using the formula for $w_{0}(R)$ given by (2.7)-(2.8) with $f(t)=\rho^{2}(t)$. From this fact we have

$$
\begin{aligned}
-\lim _{R \rightarrow \infty} \oint_{S_{R}} \frac{\partial w_{0}(\varepsilon|z|)}{\partial r} d s & =-\lim _{R \rightarrow \infty} \oint_{S_{\varepsilon}} \frac{\partial w_{0}(|z|)}{\partial r} d s \\
& =-\left.\lim _{R \rightarrow \infty} \int_{0}^{2 \pi} \frac{\partial w_{0}(r)}{\partial r}\right|_{r=R} R d \theta \\
& =-2 \pi \lim _{R \rightarrow \infty} R \frac{\partial w_{0}(R)}{\partial R} \\
& =2 \pi \int_{0}^{\infty} \varphi_{0}(t) t \rho^{2}(t) d t>0
\end{aligned}
$$

where the estimate on $I$ in the proof of Lemma 2.2 was used in the last equality. The formula (4.18) is proved. Finally let us prove (4.19). By the divergence theorem

$$
\begin{aligned}
\lim _{R \rightarrow \infty} \oint_{S_{R}} \frac{\partial u_{\varepsilon}^{*}(\varepsilon x)}{\partial r} d s & =\lim _{R \rightarrow \infty} \oint_{S_{\varepsilon R}} \frac{\partial u_{\varepsilon}^{*}(x)}{\partial r} d s \\
& =\lim _{R \rightarrow \infty} \int_{B_{\varepsilon R}} \Delta u_{\varepsilon}^{*}(x) d x=\int_{\mathbb{R}^{2}} \Delta u_{\varepsilon}^{*}(x) d x
\end{aligned}
$$


and

$$
\begin{aligned}
\left|\int_{\mathbb{R}^{2}} \Delta u_{\varepsilon}^{*}(x) d x\right| & \leq \int_{\mathbb{R}^{2}}\left|\Delta u_{\varepsilon}^{*}(x)\right| d x \\
& =\int_{\mathbb{R}^{2}}\left|\Delta u_{\varepsilon}^{*}(x)\right|\left(1+|x|^{\frac{17}{8}}\right)^{\frac{1}{2}}\left(1+|x|^{\frac{17}{8}}\right)^{-\frac{1}{2}} d x \\
& \leq\left(\int_{\mathbb{R}^{2}}\left|\Delta u_{\varepsilon}^{*}(x)\right|^{2}\left(1+|x|^{\frac{17}{8}}\right) d x\right)^{\frac{1}{2}}\left(\int_{\mathbb{R}^{2}} \frac{d x}{1+|x|^{\frac{17}{8}}}\right)^{\frac{1}{2}} \\
& \leq C\left\|u_{\varepsilon}^{*}\right\|_{Y_{\frac{1}{8}}} \leq C\left\|v_{\varepsilon}^{*}\right\|_{U_{\frac{1}{8}}} \leq C \varepsilon^{3}
\end{aligned}
$$

where (4.2) was used in the last inequality. We thus proved (4.19). The proof of the theorem is complete.

Remark 4.1. Our method of construction of solutions of the problem (9), (10) actually provides an explicit formula for approximation of those solutions. Indeed, the proof of Theorem 2.1 is based on the following modified version of Newton's iteration method:

$$
v_{n+1}=v_{n}-\left[\mathbf{P}_{\varepsilon}^{\prime}\left(v_{0}\right)\right]^{-1} \mathbf{P}_{\varepsilon}\left(v_{n}\right)
$$

In our case the stating point is $v_{0}=0 \in U_{\frac{1}{8}}$ and the functions $\rho_{\varepsilon, a_{\varepsilon}^{*}}, w_{0}(x)$ are given by explicit formula. It is well known (see [12]) that the above iteration scheme has exponential rate of convergence.

\section{APPENDIX}

Proof of Lemma 2.1. Let $w(r)$ be a solution to $L_{1} w=f$. Since $\varphi_{0}(r)$ is a solution of (2.6) with $f=0$, we try ansatz $w(r)=\xi(r) \varphi_{0}(r)$, and obtain $\xi(r)$ below. By an elementary computation we have

$$
f=L_{1} w=\varphi_{0} \xi^{\prime \prime}+\left(2 \varphi_{0}^{\prime}+\frac{\varphi_{0}}{r}\right) \xi^{\prime}
$$

where we used the fact $L_{1} \varphi_{0}=0$. Multiplying the above equations by $r \varphi_{0}$, we deduce that

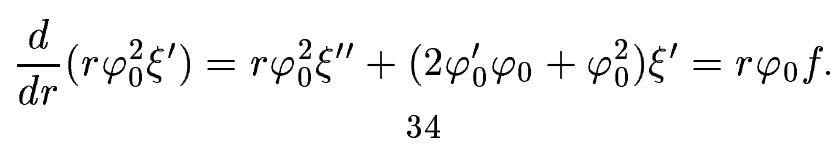


From this we immediately have

$$
\begin{aligned}
\xi(r) & =\int_{0}^{r} \frac{1}{s \varphi_{0}^{2}(s)}\left\{\int_{0}^{s} \varphi_{0}(t) t f(t) d t\right\} d s \\
& =\int_{0}^{r} \frac{\phi_{f}(s)}{(1-s)^{2}} d s, \quad r \in(0,1)
\end{aligned}
$$

where $\phi_{f}(\cdot)$ is defined by

$$
\phi_{f}(s)=\frac{(s-1)^{2}}{s \varphi_{0}^{2}(s)} \int_{0}^{s} t f(t) \varphi_{0}(t) d t \quad s \in(0, \infty)
$$

as in (2.8).

Thus, for $r \in(0,1)$ we find that

$$
w_{1}(r)=\varphi_{0}(r) \int_{0}^{r} \frac{\phi_{f}(s)}{(1-s)^{2}} d s
$$

solves $(2.6)$ for $r \in(0,1)$. Similarly we can deduce that for any $\epsilon>0$

$$
w_{2}(r)=\varphi_{0}(r) \int_{1+\epsilon}^{r} \frac{\phi_{f}(s)}{(1-s)^{2}} d s
$$

solves (2.6) for $r \in(1, \infty)$.

In order to extend our solution $w(r)$ to the range $r \in(0, \infty)$ we set

$$
\begin{aligned}
w(r) & =\varphi_{0}(r) \int_{0}^{r} \frac{\phi_{f}(s)-\phi_{f}(1)}{(1-s)^{2}} d s+\varphi_{0}(r) \frac{\phi_{f}(1) r}{1-r} \\
& =I_{1}(r)+I_{2}(r)
\end{aligned}
$$

We claim that $w(r)$ ia a well-defined $C_{l o c}^{2}\left(\mathbb{R}_{+}^{1}\right)$ solution of $(2.6)$.

Obviously $I_{2} \in C_{\text {loc }}^{\infty}\left(\mathbb{R}_{+}^{1}\right)$. In order to show that $I_{1} \in C_{\text {loc }}^{2}\left(\mathbb{R}_{+}^{1}\right)$ we observe first $\phi_{f}(s) \in C_{l o c}^{1}\left(\mathbb{R}_{+}^{1}\right), \phi_{f}(s) \in C^{2}(0, k)$ for all $k>0$. In particular, we have

$$
\lim _{s \rightarrow 0+} \frac{1}{s} \int_{0}^{s} \varphi_{0}(t) t f(t) d t=0
$$

and there is no singularity near $s=0$ of $\phi_{f}(s)$. Next we will show that

$$
\phi_{f}^{\prime}(1)=0 \text {. }
$$


To see this we compute first

$$
\begin{aligned}
\phi_{f}^{\prime}(s) & =\frac{(s-1) \eta(s)}{s^{2} \varphi_{0}^{3}(s)} \int_{0}^{s} t f(t) \varphi_{0}(t) d t+\frac{(1-s)^{2} f(s)}{\varphi_{0}(s)} \\
& =J_{1}(s)+J_{2}(s)
\end{aligned}
$$

where we set

$$
\eta(s)=(s+1) \varphi_{0}(s)-2(s-1) s \varphi_{0}^{\prime}(s) .
$$

We note $J_{2}(1)=0$. In order to show $J_{1}(1)=0$ it suffices to prove

$$
\eta(1)=\eta^{\prime}(1)=\eta^{\prime \prime}(1)=0
$$

Obviously $\eta(1)=0$. Since

$$
\eta^{\prime}(s)=\varphi_{0}(s)-3(s-1) \varphi_{0}^{\prime}(s)-2(s-1) s \varphi_{0}^{\prime \prime}(s)
$$

we also find that $\eta^{\prime}(1)=0$. In order to see $\eta^{\prime \prime}(1)=0$ we use substitution $s \varphi_{0}^{\prime \prime}=-\varphi_{0}^{\prime}-s \rho(s) \varphi_{0}$ for the last term in the right hand side of (A.4), which is immediate from the ordinary differential equation for $\varphi_{0}, L_{1} \varphi_{0}=0$. Thus, we obtain

$$
\begin{aligned}
\eta^{\prime}(s) & =\varphi_{0}(s)-3(s-1) \varphi_{0}^{\prime}(s)+2(s-1)\left(\varphi_{0}^{\prime}+s \rho \varphi_{0}\right) \\
& =\varphi_{0}(s)-(s-1) \varphi_{0}^{\prime}(s)+2 s(s-1) \rho(s) \varphi_{0}(s)
\end{aligned}
$$

from which $\eta^{\prime \prime}(1)=0$ follows easily. We thus proved (A.3), and hence (A.2).

From (A.2) we have

$$
\frac{\phi_{f}(s)-\phi_{f}(1)}{(1-s)^{2}} \in C^{1}(0, k) \quad \forall k>0 .
$$

This implies $I_{1}(r)$ in (A.1), and hence $w(r)$ belongs to $C^{2}(0, k)$ for all $k>0$. Now, in order to show that $w(r)$ is a $C^{2}$ solution of $(2.6)$ in $(0, \infty)$, it suffices to prove that $w(r)$ satisfies $(2.6)$ on $(0,1)$ and $(1, \infty)$. For $r \in(0,1)$ we find easily

$$
w(r)=\varphi_{0}(r) \int_{0}^{r} \frac{\phi_{f}(s)}{(1-s)^{2}} d s=w_{1}(r),
$$

which is a solution of (2.6) by construction. 
For $r>1$, setting $\epsilon>0$, we have

$$
\begin{aligned}
w(r) & =\varphi_{0}(r)\left\{\int_{1+\epsilon}^{r} \frac{\phi_{f}(s)-\phi_{f}(1)}{(1-s)^{2}} d s+\int_{0}^{1+\epsilon} \frac{\phi_{f}(s)-\phi_{f}(1)}{(1-s)^{2}} d s+\frac{\phi_{f}(1) r}{1-r}\right\} \\
& =w_{2}(r)+\varphi_{0}(r)\left\{-\frac{(1+\epsilon) \phi_{f}(1)}{\epsilon}+\int_{0}^{1+\epsilon} \frac{\phi_{f}(s)-\phi_{f}(1)}{(1-s)^{2}} d s\right\} .
\end{aligned}
$$

By construction the first term above is a solution of (2.6), while the second term is solution of the homogeneous equation of (2.6)(i.e. (2.6) with $f=0$ ).

This completes the proof of Lemma 2.1.

Proof of Lemma 2.2. From the explicit solution formula (2.8) we find that $w_{0}(\cdot) \in C^{0}\left(\mathbb{R}_{+}\right)$, and thus (2.9) follows from (2.10). It suffices now to prove (2.10). We observe from formula (2.8) that

$$
w_{0}(r)=\varphi_{0}(r) \int_{2}^{r}\left(\frac{1+s^{2 N+2}}{1-s^{2 N+2}}\right)^{2} \frac{I(s)}{s} d s+(\text { bounded function of } r)
$$

as $r \rightarrow \infty$, where

$$
I(s)=\int_{0}^{s} \varphi_{0}(t) t \rho^{2}(t) d t
$$

Since $\varphi_{0}(r) \rightarrow-1$ as $r \rightarrow \infty,(2.10)$ follows if

$$
I=I(\infty)=\int_{0}^{\infty} \varphi_{0}(r) r \rho^{2}(r) d r>0 .
$$

Indeed, substituting $r^{2}=t$ in the integrand of $I$, then

$$
\begin{array}{r}
I=32(N+1)^{4} \int_{0}^{\infty}\left(\frac{1-t^{N+1}}{1+t^{N+1}}\right) \frac{t^{2 N}}{\left(1+t^{N+1}\right)^{4}} d t \\
=32(N+1)^{4} \int_{0}^{1} \frac{\left(1-t^{N+1}\right) t^{2 N}}{\left(1+t^{N+1}\right)\left(1+t^{N+1}\right)^{4}} d t \\
\quad+32(N+1)^{4} \int_{1}^{\infty} \frac{\left(1-t^{N+1}\right) t^{2 N}}{\left(1+t^{N+1}\right)\left(1+t^{N+1}\right)^{4}} d t \\
=32(N+1)^{4} \int_{0}^{1} \frac{\left(1-t^{N+1}\right) t^{2 N}}{\left(1+t^{N+1}\right)\left(1+t^{N+1}\right)^{4}} d t \\
\quad-32(N+1)^{4} \int_{0}^{1} \frac{\left(1-s^{N+1}\right) s^{2 N+2}}{\left(1+s^{N+1}\right)\left(1+s^{N+1}\right)^{4}} d s \\
=32(N+1)^{4} \int_{0}^{1} \frac{\left(1-t^{N+1}\right)\left(1-t^{2}\right) t^{2 N}}{\left(1+t^{N+1}\right)\left(1+t^{N+1}\right)^{4}} d t>0
\end{array}
$$


where we changed variable $s=\frac{1}{t}$ in the third equality.

Proof of Lemma 2.5. Suppose $L_{1}$ be the differential operator introduced in (2.6). Let us first observe that the following equality holds.

$$
L_{1}\left[\frac{1}{16\left(1+r^{2 N+2}\right)^{2}}\right]=\frac{(N+1)^{2} r^{4 N+2}}{\left(1+r^{2 N+2}\right)^{4}},
$$

which can be verified by an elementary computation. We prove our lemma for $\varphi_{+}$only. The case for $\varphi_{-}$is similar. Using the above identity, we have the following

$$
\begin{aligned}
& \int_{\mathbb{R}^{2}}\left(\rho w_{0}-2 \rho^{2}\right) \varphi_{+}^{2} d x=\int_{0}^{2 \pi} \int_{0}^{\infty}\left(\rho w_{0}-2 \rho^{2}\right) \frac{r^{2 N+2} \cos ^{2}(N+1) \theta}{\left(1+r^{2 N+2}\right)^{2}} r d r d \theta \\
& =\int_{0}^{2 \pi} \cos ^{2}(N+1) \theta d \theta \int_{0}^{\infty}\left[\frac{8(N+1)^{2} r^{2 N}}{\left(1+r^{2 N+2}\right)^{2}} w_{0}-2 \rho^{2}\right] \frac{r^{2 N+2}}{\left(1+r^{2 N+2}\right)^{2}} r d r \\
& =\pi \int_{0}^{\infty}\left\{\frac{1}{2} L_{1}\left[\frac{1}{\left(1+r^{2 N+2}\right)^{2}}\right] w_{0}-\frac{2 \rho^{2} r^{2 N+2}}{\left(1+r^{2 N+2}\right)^{2}}\right\} r d r \\
& =\pi \int_{0}^{\infty}\left\{\frac{1}{2} L_{1} w_{0} \cdot \frac{1}{\left(1+r^{2 N+2}\right)^{2}}-\frac{2 \rho^{2} r^{2 N+2}}{\left(1+r^{2 N+2}\right)^{2}}\right\} r d r \\
& =\pi \int_{0}^{\infty}\left\{\frac{\rho^{2}}{2\left(1+r^{2 N+2}\right)^{2}}-\frac{2 \rho^{2} r^{2 N+2}}{\left(1+r^{2 N+2}\right)^{2}}\right\} r d r \\
& =\pi \int_{0}^{\infty}\left\{\frac{5 \rho^{2}}{2\left(1+r^{2 N+2}\right)^{2}}-\frac{2 \rho^{2}}{\left(1+r^{2 N+2}\right)}\right\} r d r \\
& =64 \pi(N+1)^{4} \int_{0}^{\infty}\left\{\frac{5 r^{4 N}}{2\left(1+r^{2 N+2}\right)^{6}}-\frac{2 r^{4 N}}{\left(1+r^{2 N+2}\right)^{5}}\right\} r d r \\
& \left.=32 \pi(N+1)^{4} \int_{0}^{\infty}\left\{\frac{5 t^{2 N}}{2\left(1+t^{N+1}\right)^{6}}-\frac{2 t^{2 N}}{\left(1+t^{N+1}\right)^{5}}\right\} d t \quad \text { (By change of variable, } t=r^{2}\right) \\
& =32 \pi(N+1)^{4} \int_{0}^{\infty}\left\{\frac{-5 t^{N}}{10(N+1)} \frac{d}{d t} \frac{1}{\left(1+t^{N+1}\right)^{5}}+\frac{t^{N}}{2(N+1)} \frac{d}{d t} \frac{1}{\left(1+t^{N+1}\right)^{4}}\right\} d t \\
& =32 \pi(N+1)^{3} N \int_{0}^{\infty}\left\{\frac{t^{N-1}}{2\left(1+t^{N+1}\right)^{5}}-\frac{t^{N-1}}{2\left(1+t^{N+1}\right)^{4}}\right\} d t \\
& =-16 \pi(N+1)^{3} N \int_{0}^{\infty} \frac{t^{2 N}}{\left(1+t^{N+1}\right)^{5}} d t<0 .
\end{aligned}
$$

The proof of the lemma is completed. 
Proof of Lemma 3.1. We write $g_{\varepsilon}(z, a)$ as follows

$$
\begin{aligned}
& g_{\varepsilon}(z, a)=\frac{r^{2 N}+\varepsilon h_{1}(z)}{\left(1+\left|z^{N+1}+\varepsilon h_{2}(z)+\frac{a}{\varepsilon^{2}}\right|^{2}\right)^{2}} \\
& =\frac{\varepsilon^{8} r^{2 N}+\varepsilon^{9} h_{1}(z)}{\left(\varepsilon^{4}+\varepsilon^{4} r^{2 N+2}+|a|^{2}+2 \varepsilon^{2} \operatorname{Re}\left\{\bar{a} z^{N+1}\right\}+2 \varepsilon^{3} \operatorname{Re}\left\{\bar{a} h_{2}(z)\right\}+\varepsilon^{5} h_{3}(z)\right)^{2}},
\end{aligned}
$$

where

$$
\begin{aligned}
& \left|h_{1}(z)\right| \leq C\left(1+|z|^{2 N-1}\right) \\
& \left|h_{2}(z)\right| \leq C\left(1+|z|^{N}\right) \\
& \left|h_{3}(z)\right| \leq C\left(1+|z|^{2 N+1}\right) .
\end{aligned}
$$

There exists $R_{0}>1, \varepsilon_{0} \in(0,1)$ such that $|z|=r>R_{0}$ and $|a|<\varepsilon^{2}$, $\varepsilon \in\left(0, \varepsilon_{0}\right)$ imply

$$
\begin{aligned}
2 \varepsilon^{2}|a| r^{N+1} & \leq 2 \varepsilon^{4} r^{N+1} \leq \frac{\varepsilon^{4}}{4} r^{2 N+2} \\
2 \varepsilon^{3}|a|\left|h_{2}(z)\right| & \leq C \varepsilon^{5}\left(1+|z|^{N}\right) \leq \frac{3}{8} \varepsilon^{4}+\frac{1}{4} \varepsilon^{4} r^{2 N+2} \\
\varepsilon^{5}\left|h_{3}(z)\right| & \leq C \varepsilon^{5}\left(1+|z|^{2 N+1}\right) \leq \frac{3}{8} \varepsilon^{4}+\frac{1}{4} \varepsilon^{4} r^{2 N+2} .
\end{aligned}
$$

Substituting these estimates into the formula (A.5), we obtain

$$
\begin{aligned}
\left|g_{\varepsilon}(z, a)\right| & \leq \frac{C \varepsilon^{8} r^{2 N}}{\left(\varepsilon^{4}+\varepsilon^{4} r^{2 N+2}+|a|^{2}-2 \varepsilon^{2}|a| r^{N+1}-2 \varepsilon^{3}|a|\left|h_{2}(z)\right|-\varepsilon^{5}\left|h_{3}(z)\right|\right)^{2}} \\
& \leq \frac{C \varepsilon^{8} r^{2 N}}{\left(\frac{\varepsilon^{4}}{4}+\frac{\varepsilon^{4}}{4} r^{2 N+2}\right)^{2}} \leq \frac{C r^{2 N}}{\left(1+r^{2 N+2}\right)^{2}}
\end{aligned}
$$

for all sufficiently large $r$. Below we put

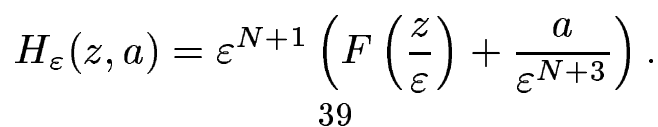


To estimate $\frac{\partial g_{\varepsilon}(z, a)}{\partial a_{j}}$ and $\frac{\partial^{2} g_{\varepsilon}(z, a)}{\partial a_{j} \partial a_{k}}$ we compute as follows.

$$
\begin{aligned}
\frac{\partial g_{\varepsilon}(z, a)}{\partial a_{1}} & =-\frac{4}{\varepsilon^{2}} g_{\varepsilon}(z, a) \frac{\operatorname{Re} H_{\varepsilon}(z, a)}{1+\left|H_{\varepsilon}(z, a)\right|^{2}}, \\
\frac{\partial g_{\varepsilon}(z, a)}{\partial a_{2}} & =-\frac{4}{\varepsilon^{2}} g_{\varepsilon}(z, a) \frac{\operatorname{Im} H_{\varepsilon}(z, a)}{1+\left|H_{\varepsilon}(z, a)\right|^{2}}, \\
\frac{\partial^{2} g_{\varepsilon}(z, a)}{\partial a_{1}^{2}} & =-\frac{4}{\varepsilon^{4}} \frac{g_{\varepsilon}(z, a)}{1+\left|H_{\varepsilon}(z, a)\right|^{2}}+\frac{24}{\varepsilon^{4}} g_{\varepsilon}(z, a) \frac{\left(\operatorname{Re} H_{\varepsilon}(z, a)\right)^{2}}{\left(1+\left|H_{\varepsilon}(z, a)\right|^{2}\right)^{2}}, \\
\frac{\partial^{2} g_{\varepsilon}(z, a)}{\partial a_{1} \partial a_{2}} & =\frac{24}{\varepsilon^{4}} g_{\varepsilon}(z, a) \frac{\operatorname{Re} H_{\varepsilon}(z, a) \operatorname{Im} H_{\varepsilon}(z, a)}{\left(1+\left|H_{\varepsilon}(z, a)\right|^{2}\right)^{2}} \\
\frac{\partial^{2} g_{\varepsilon}(z, a)}{\partial a_{2}^{2}} & =-\frac{4}{\varepsilon^{4}} \frac{g_{\varepsilon}(z, a)}{1+\left|H_{\varepsilon}(z, a)\right|^{2}}+\frac{24}{\varepsilon^{4}} g_{\varepsilon}(z, a) \frac{\left(\operatorname{Im} H_{\varepsilon}(z, a)\right)^{2}}{\left(1+\left|H_{\varepsilon}(z, a)\right|^{2}\right)^{2}} .
\end{aligned}
$$

Since

$$
\frac{\left|H_{\varepsilon}(z, a)\right|}{1+\left|H_{\varepsilon}(z, a)\right|^{2}} \leq \frac{1}{2}
$$

we have

$$
\sum_{i=1}^{2}\left|\frac{\partial g_{\varepsilon}(z, a)}{\partial a_{j}}\right| \leq \frac{4}{\varepsilon^{2}}\left|g_{\varepsilon}(z, a)\right| \leq \frac{C r^{2 N}}{\varepsilon^{2}\left(1+r^{2 N+2}\right)^{2}},
$$

and

$$
\sum_{j, k=1}^{2}\left|\frac{\partial^{2} g_{\varepsilon}(z, a)}{\partial a_{j} \partial a_{k}}\right| \leq \frac{C}{\varepsilon^{4}}\left|g_{\varepsilon}(z, a)\right| \leq \frac{C r^{2 N}}{\varepsilon^{4}\left(1+r^{2 N+2}\right)^{2}}
$$

for $|z| \geq R_{0}$. This completes the proof of the lemma.

\section{REFERENCES}

1. S. Baraket and F. Pacard, Construction of singular limits for a semilinear elliptic equation in dimension 2, Calc. Var. and P.D.E. 6 (1998), 1-38.

2. L. Caffarelli and Y. Yang, Vortex condensation in the Chern-Simons-Higgs model:an existence theory, Comm. Math. Phys. 168 (1995), 321-336.

3. D. Chae and N. Kim, Topological multivortex solutions of the self-dual Maxwell-ChernSimons-Higgs System, J. Diff. Eqns 134 No.1 (1997), 154-182.

4. X. Chen, S. Hastings, J. B. McLeod, and Y. Yang, A nonlinear elliptic equations arising from gauge field theory and cosmology, Proc. R. Soc. Lond. A. 446 (1994), 453-478.

5. G. Dunne, "Self-Dual Chern-Simons Theories", Lecture Notes in Physics, vol. M36, Springer -Verlag, Berlin, New York, 1995. 
6. A. V. Fursikov and O. Yu. Imanuvilov, Local Exact Boundary Controllability of the Boussinesq Equation, SIAM Journal of Control and Optimization 36 No. 2 (1998), 391-421.

7. D. Gilbag and N.S.Trudinger, "Elliptic Partial Differential Equations of Second order"2nd ed., Springer-Verlag, New York, 1982.

8. J. Hong, Y. Kim, and P.Y. Pac, Phys. Rev. Lett. 64 (1990), 2230.

9. R. Jackiw and S. Y. Pi, Phys. Rev. Lett. 64 (1990), 2969.

10. R. Jackiw and E. J. Weinberg, Phys. Rev. Lett. 63 (1990), 2234.

11. A. Jaffe and C. Taubes, Vortices and Monopoles (1980), Birkhäuser, Boston.

12. L.V. Kantorovich and G.P. Akilov, Functional Analysis 2nd ed., Pergamon Press, 1982.

13. J. Spruck and Y. Yang, Topological solutions in the self-dual Chern-Simons theory: Existence and approximation, Ann. Inst. Henri Poincaré 1 (1995), 75-97.

14. J. Spruck and Y. Yang, The existence of nontopological solitons in the self-dual ChernSimons theory, Comm. Math. Phys. 149 (1992), 361-376.

15. G. Tarantello, Multiple condensate solutions for the Chern-Simons-Higgs Theory, J. Math. Phys. 37 (1996), 3769-3796.

16. R. Wang, The existence of Chern-Simons Vortices, Comm. Math. Phys. 137 (1991), 587-597.

1 Department of Mathematecs, Seoul National University, Seoul 151-742, KOREA

E-mail address: dhchae@math.snu.ac.kr

2 Department of Mathematics, Iowa State University, Ames, iA 50011, USA

E-mail address: vika@iastate.edu 